\title{
Lasers, photon statistics, photon-correlation spectroscopy and subsequent applications
}

\author{
Roy Pike \\ roy.pike@kcl.ac.uk \\ King's College London, Strand, London, WC2R 2LS, United Kingdom
}

A review is given of developments in the theory and application of the quantum statistics of visible-light photons, particularly of scattered laser light, over the fifty years since Maiman's publication of the first working laser. Some introductory pre-laser historical background to photon statistics is included and details are given of research and commercial developments in the field conducted in the author's laboratory in the UK over the period. The resulting emergence of photon statistics and photon-correlation spectroscopy as widely used techniques for non-invasive measurements of diffusion and velocity in many present-day areas of science and industry is described. [DOI: 10.2971/jeos.2010.10047S]

Keywords: photon statistics, laser scattering, nanosizing, velocimetry

\section{INTRODUCTION AND OVERVIEW}

It is a great pleasure for me to be invited to contribute to this volume, celebrating the first fifty years of the laser, to recount in a discursive and somewhat personal manner our work on the statistics of photon detections of coherent and scattered laser light and the transition of this work from blue-skies research to a substantial continuing worldwide industry.

The study of the statistics of visible and infrared radiation all derived from Planck's phenomenological introduction of the quantum of energy at the turn of the last century [1,2]. He argued, as an "act of desperation" 1 that each field mode in a black-body cavity could only receive energy from the cavity-wall oscillators in discrete amounts. He did not, at this time or later, wish to suggest that the radiation field was quantised. It was Einstein, a master of thermodynamics, fluctuations and statistics who, using these skills to great effect in pioneering (and Nobel prize-winning) work [4], on the statistics of incoherent radiation fields in thermal equilibrium with their sources, took the historic step of endowing the light field itself with energy quanta and thus gave birth to the photon and its statistical properties.

As an experimental discipline, although we shall describe some subsequent important pre-laser experiments on intensity statistics and correlations of incoherent light sources in the mid century, the typical sub-nanosecond inverse linewidths of thermal equilibrium fields made these difficult and specialised. For many decades after Einstein's Nobel prize in 1921 there was little motivation for discussing the possibility of non-equilibrium radiation, the advent of which was destined to change this situation. Indeed, apart from a few works on inverting the population of two energy levels, by Ladenburg in 1928 [5], Fabrikant in 1940 [6], (see p. 113 of [7]) and Kastler's Nobel prize winning work with Brossel on optical pumping from 1949 [8], this was not to come in any detailed fashion until the invention of the maser and laser ${ }^{2}$. The first laser was soon followed

\footnotetext{
${ }^{1}$ See letter to R W Wood [3]

${ }^{2}$ See Bertolotti's well known history of the laser [7].
}

by the pioneering (and also Nobel prize-winning) theoretical papers of Roy Glauber in 1963 [9]- [11], in which a full quantum theory of coherent and more general non-equilibrium photon statistics and correlations was finally presented.

Glauber's predictions of Poisson photon-counting distributions for coherent laser light opposed Bose-Einstein and their limiting geometric distributions for incoherent light and his work became the object of considerable further study, both theoretically and experimentally. We shall mention several preliminary tests of these predictions and innovative laser scattering experiments made in the following years using conventional analogue pulse integration techniques. However, coincidentally, and fortunately, 1963 was also the year when Thomas Longo [12], after a patent of Buie [13], led the design of the first TTL (Transistor Transistor Logic) family, Sylvania Universal High-level Logic (SUHL), which was soon followed by the commercial availability of medium and large-scale silicon integrated circuits with a typical gate propagation delay of $10 \mathrm{~ns}$.

In the year following Glauber's papers, with some care in selecting and developing single-photon detectors and circuits, we were able to marry them with quantities of fast silicon integrated transistor circuits and were thus able to accumulate highly accurate digital photon-counting statistics and, subsequently, digital photoncorrelation functions, in both the incoherent and coherent limits of light fields. Of course, at that time, great care was also needed in the choice of high-quality laser sources to investigate for the coherent limit. For incoherent light, although at first, following the pre-laser experiments, we used a narrow mercury line source, eventually much narrower linewidths were generated by scattering coherent laser beams from randomly diffusing Brownian motion of macromolecules in solution. These experiments confirmed not only the basic Poisson and geometric distributions at short sample times for coherent and incoherent light, respectively, but opened up the whole gamut of integrated distributions for incoherent sources and lasers through threshold, as theoretical results from our own calcu- 


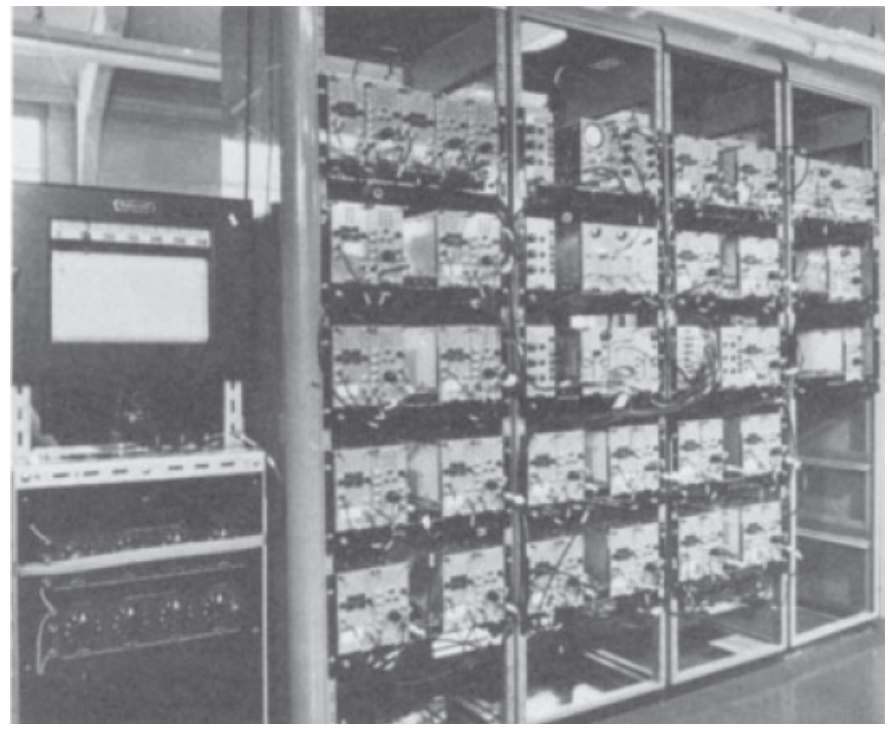

FIC. 1 The original apparatus at AERE Harwell in 1964.

lations and those of others became available. We shall discuss some of these results later.

Our first experiments were carried out by making use of large quantities of fast nuclear electronic equipment kindly loaned to us by Atomic Energy Research Establishment at Harwell and our experimental apparatus was first set up there. We had helped them in similar ways in their early days and they were happy to make a quid pro $q u o$ in this way. The kit was eventually moved to Malvern and about this time Eric Jakeman, a theoretical physicist, and Chris Oliver, an experimentalist, came on the scene. They, together with Robin Jones, an apprentice in electonics at the time (and worth his weight in gold) and myself, pushed on the research at a rapid pace. A photograph of the apparatus at Harwell circa 1964 is shown in Figure 1. Our first theoretical paper on the photon counting statistics of gaussian light [14] appeared in 1965 and our first report on these experiments was presented at the International Conference on the Physics of Quantum Electronics in Puerto Rico in the same year [15]. In the latter, with some preliminary results on a mercury line, we also outlined some of the technical problems of this challenging new technique.

Rapid dissemination and use of the methods produced by our research took place not only by the usual channels of scientific publication and conferences but they quickly turned out to have patentable practical applications in the spectroscopy of intensity fluctuations of scattered laser beams. The apparatus was duly commercialised, using the new TTL integrated circuits, as the "Malvern Correlator" in 1971. Licensing of the patent was undertaken by the UK National Research and Develoment Corporation on behalf of the government. This unit, shown in Figure 2, went to many laboratories around the world in the years following. It was named K7023 by the company chairman after one of his favourite steam railway engines! Although we did not know at the time, I was to say later in a Charles Parsons (of naval turbine fame) memorial lecture in 1976 [16], “... through the macromolecular range of viruses, proteins and polymers, right into that of macroscopic bodies, such as, for instance, aeroplanes and tanks... almost anything that diffuses, shakes, vibrates, translates, rotates or continues moving in any way at all is fair game for an experiment in photon correlation". With suitable laser optics all the

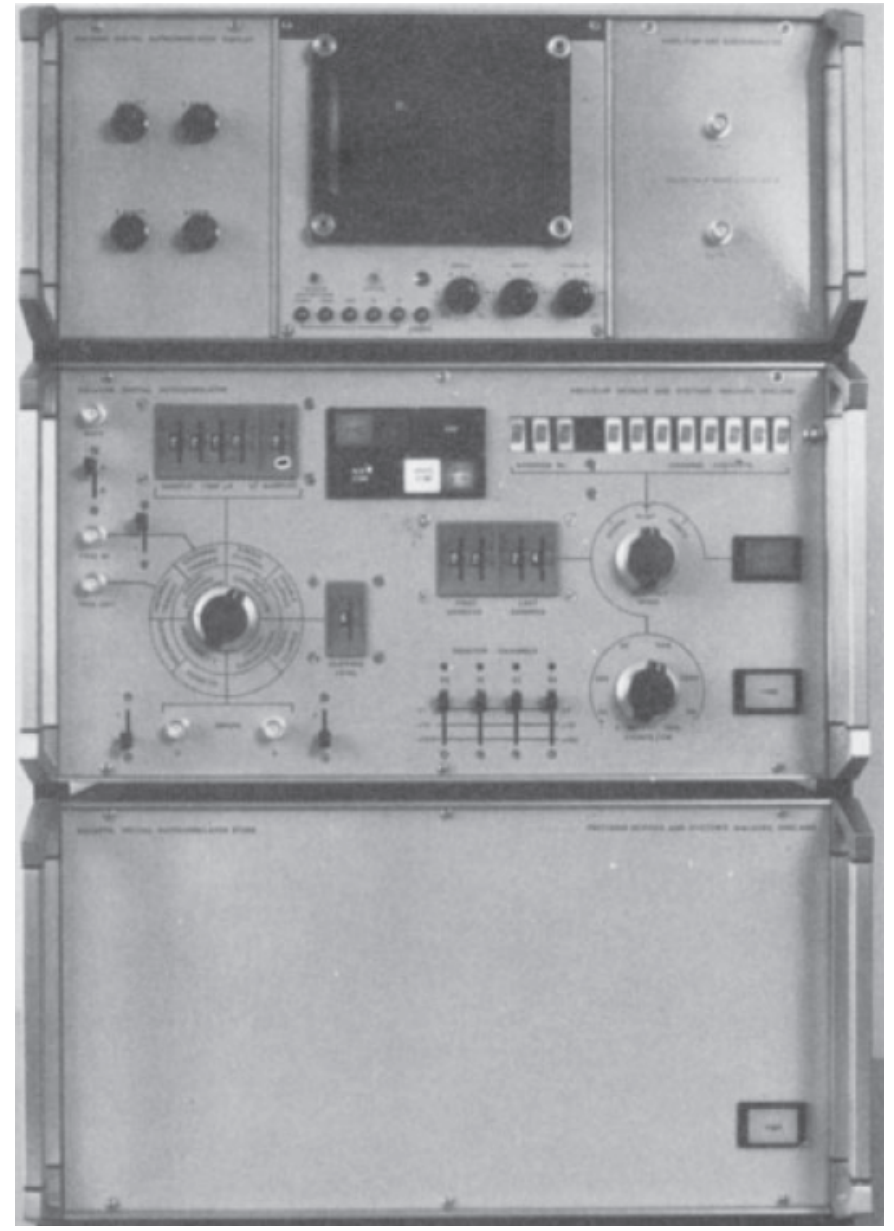

FIG. 2 The first commercial Malvern Correlator, 1971 (K7023).

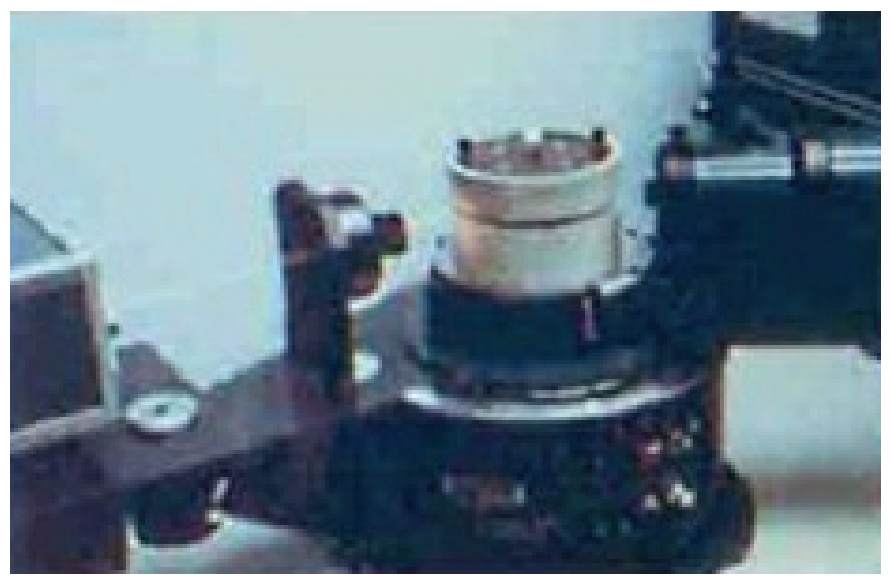

FIG. 3 An early laser scattering goniometer.

motion frequencies in the intensity fluctuation spectrum of the scattered light show up in accurate, convenient and interpetable ways in the photon-correlation function and its Fourier transform. For particle sizing the electronics had to be paired up with a laser-scattering goniometer such as is depicted in Figure 3.

The scientific communication, I should say, was aided considerably by awards from the NATO Scientific Affairs Commission to conduct Advanced Study Institutes in Capri in Italy in 1973 [17], and 1976 [18], (both co-directed with Hermann Cummins, now at NYU, and with the invaluable help of Mario Bertolotti, the present editor of this Journal, who has a beautiful home there). We met many of 

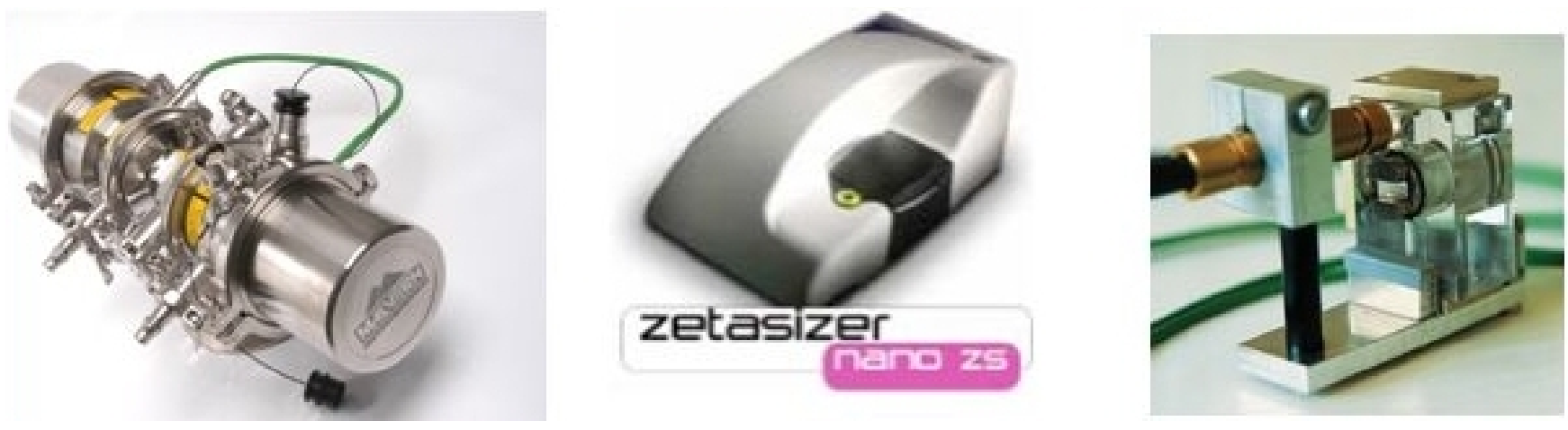

FIG. 4 Some recent Malvern particle-sizing equipment. Left to right: On-line particle sizing, nano-particle sizing and European space agency particle-sizing optrode.

our later lifetime colleagues and leaders in the fields of "Photoncounting statistics" and "Photon-correlation spectroscopy" at these summer schools. These have been followed by a series of approximately biennial international gatherings on such questions in quantum optics with generous help over the years from a number of bodies including particularly NASA, ONR (European Office) and OSA from the USA and from IOP and the Ministry of Defence from the UK. This series included two memorable NATO Advanced Research Workshops in 1988 in Cortina, Italy [19], co-directed with Paolo Tombesi from the University of Rome, La Sapienza and 1996 in Krakow [20], Poland co-directed with Igor Yudin from the Oil and Gas Research Institute of the Russian Academy of Sciences.

Over these years, used as a sophisticated digital optical radar, the photon statistics of scattered laser light have been applied to measure motions of many types, from the Brownian movement of micro- and nano-molecules and particles to the internal duct flow in sub- and super-sonic aeroengines.

Among its applications the Malvern Correlator became widely used as the world's first commercial, laser-based, particle-size analyzer. Photon correlation spectroscopy has now become universally adopted and pervades a number of modern scientific, industrial and medical processes, characterising, at-line, on-line and in-line, size, shape, molecular weight, chemical composition, aggregation, polydisperity and rheological properties of particles from nanometers to microns. The technique is now used for example, in polymer science, in emulsion preparation in the food industry, in the manufacture of paints and inks, in mining and geology, for catalysts and cements, for the characterisation of fogs and sprays, in water treatment, in the study of micelles in the manufacture of soaps and detergents and for abrasives and polishes. In biology and medicine the particles may be macromolecules of varying types including proteins, enzymes and viruses.

In pharmacology both water-in-oil and oil-in-water microemulsions can enhance the oral bio-availability of drugs. Submicron oil-inwater emulsions are also used for the intravenous administration of physiological water-insoluble triglycerides, the mean size and polydispersity of the particle distributions are important parameters which influence their bio-pharmaceutical fate. The method is also used to measure particle charge (zeta potential) from the Doppler shift in an electric field.

Other important applications include the measurement of flow fields in liquids and gases by following the motion of small, usually natu- ral, particles suspended in the flow, for example, for ship and aircraft design. The method provided the most sensitive method available for non-invasive measurements of flow fields and was quickly taken up by many institutions and companies worldwide. In the UK these included Rolls-Royce for improving efficiency of their aircraft engines, including the RB 211 and Olympus 593 (Concorde), British Gas for research and development of gas burners, Royal Aircraft Establishments for the design of aircraft wing profiles in their suband super-sonic wind tunnels, Admiralty Establishments for ship and submarine water channels, amongst many others. We shall show some pictures of these rigs later.

The British company Holset, for example, estimated that improvements made in this way with rigs at Cranfield to optimise blade loadings and improve wake formation in their turbochargers has reduced fuel costs by $£ 500$ per truck per year. Overseas, many companies in similar fields such as General Electric, Pratt and Whitney and Boeing followed suit. By facilitating such advances in technology it is not difficult to calculate that, over the years, this work has helped to reduce fuel costs by billions of pounds with consequent lower pollution and carbon dioxide generation.

Although our various government patents have long since expired, the original sole licencee, "Malvern Instruments Ltd" is still a major global player in particle characterisation and nanotechnology. By coincidence, as I write this paper, it has just won it's fifth "Queen's Award", a prestigeous UK industrial award for innovation, technology and export. Recent applications of their instruments in nanoscience leading to this award have been aimed at fast screening and early diagnosis of breast cancer and for studying the behaviour of proteins in research related to Alzheimer's and Parkinson's diseases. Some current products of the company are shown in Figure 4. One might note that the automated Zeta nanosizer in the centre does all and more than its ancestor shown in Figure 2, including the laser and optics shown in Figure 3!

The technique and its derivatives are variously called PhotonCorrelation Spectroscopy, Intensity-Fluctuation Spectroscopy, Laser Doppler Spectroscopy, Quasi-elastic Light Scattering and Dynamic Light Scattering and, over the years, an international community of multi-disciplinary researchers and ever more commercial vendors, a number spawned from the original manufacturer, have pushed the boundaries in countless novel directions, even reaching into outer space. 
2 PRE-GLAUBER YEARS

In 1960, the year of Maiman's first laser, I had just arrived back in the UK from a two-year Fulbright Fellowship in the Physics Department at MIT to take up a post in the scientific civil service in the theoretical physics group at the Royal Radar Establishment (RRE), Malvern. At the time this was one of the largest research establishments in physics and electronics in Europe and thus a great place to take up one's first real job.

To set the scene, I have to say that although one of my first degrees was in mathematics I did have another in physics and a PhD in experimental X-ray physics, so would regularly wear two hats, one for the desk and one for the laboratory. At MIT I moved from the hard $\mathrm{X}$-rays of my PhD years under Arthur Wilson to soft X-rays under Bert Warren. In the first case, presciently, my experiments involved counting large numbers of single photons with Geiger counters and in the second, in order to count soft X-ray photons, I had to make and activate my own photo-electron multiplier counter as a cascade of (poisonous) beryllium-copper focussing dynodes working in high vacuum. I was able to lay hands on a set of jigs for making the electron multiplier which were said to be the originals used by John Pierce and William Shockley, see Figure 5. Shockley did his PhD at MIT in 1936, and their patent was filed in 1937 so I suppose that is why they were still lying around conveniently for me at the MIT Rad. Lab. many years later. Since these formative years I have got even softer, radiation-wise, through optics to microwaves!

At RRE, with my theorist's hat, I worked first on many-body quantum mechanics, second quantisation of the general many-fermion operator, superconductivity and solid-state physics, including interband electron tunneling. A tunnel diode (Esaki diode) is a very fast semiconductor diode which uses quantum-mechanical tunneling effects. These diodes soon happened to find a crucial use in our first photomultiplier discriminator circuits.

When the papers of Glauber on quantum states of the radiation field appeared in 1963 we quickly organised a thoeretical study group to discuss their significance. Since it is massless, the photon does not have a position operator or a first quantisation so these important papers of Glauber were basically on the second-quantised many-boson operator, in fact, rather easier to deal with than the many-fermion one since the algebra is symmetric rather than Grassmann.

In 1964, not quite pre-Glauber but pre-experimental photon correlation, we should mention a first ground-breaking application of laser scattering. This the paper of Yeh and Cummins, which used the Doppler shift of a laser beam scattered from particles in a flow of fluid to measure flow velocity [21]. A little later, but also of great importance, were the papers of Ford and Benedek in 1965 on light scattering from a pure fluid near its critical point [22], of Lastovka and Benedek in 1966 on light scattering from entropy fluctuations in a normal liquid [23], and of Dubin, Lunacek and Benedek in 1967 on scattering from biological macromolecules in solution [24]. These contributions were seminal original papers using "light-beating" spectroscopy, the fore-runner of our experiments in photon-correlation spectroscopy with the same physical aims. Critical scattering from xenon, in the USMP-2 payload on the Space Shuttle flight STS-62, March 1995, (the ZENO experiment) by then of course using digital photon-correlation spectroscopy, was later to

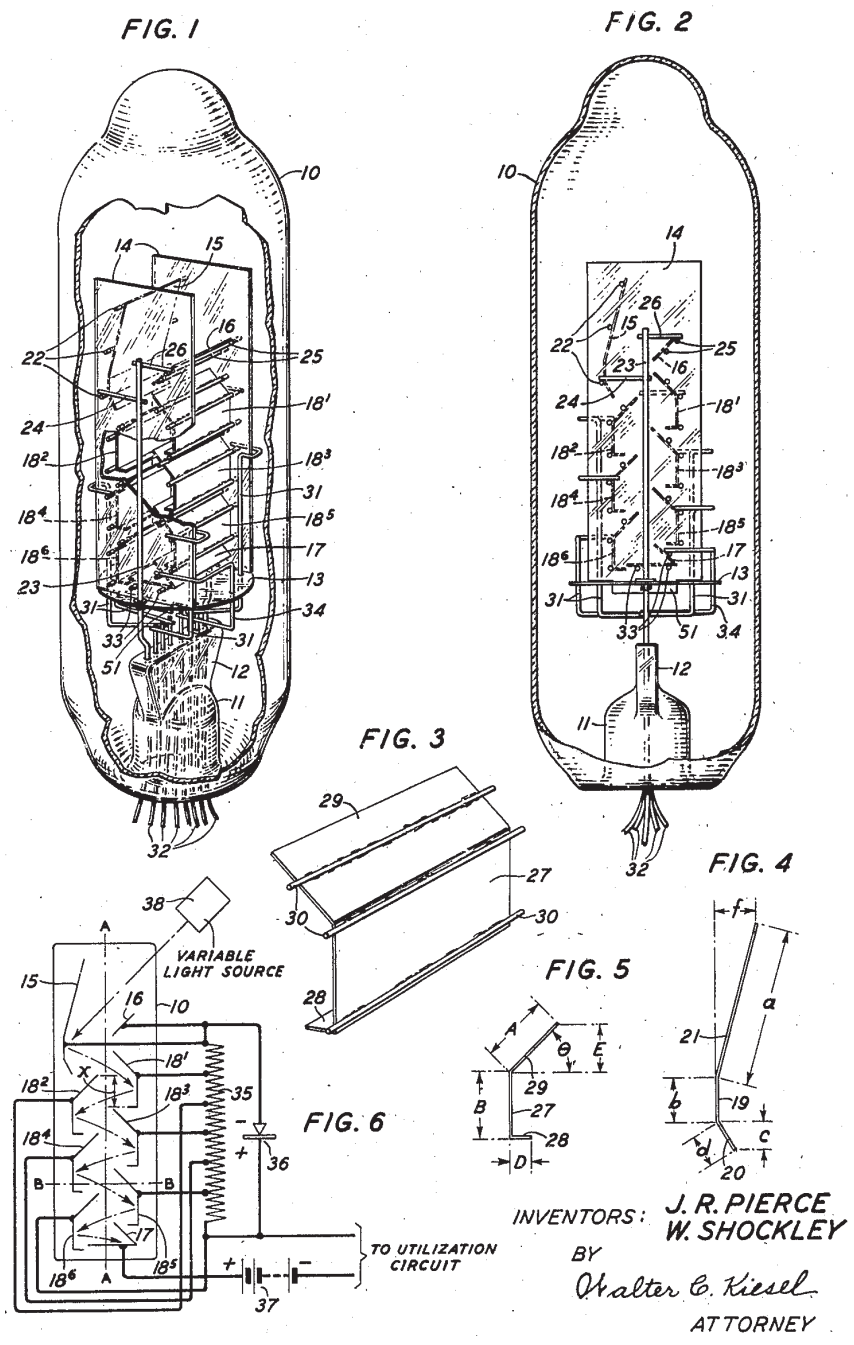

FIG. 5 The Pierce-Shockley electron multiplier.

occupy Bob Gammon of the University of Maryland and his colleagues successfully for a number of years.

\section{OPTICS, STATISTICS AND THE ORIGINS OF THE QUANTUM THEORY}

As we have mentioned, the origins of the quantised photon lay in Planck's black-body radiation law of 1900, which followed from his hypothesis that, for free radiation, the energy of an electromagnetic cavity mode of frequency $v \mathrm{~Hz}$ was dependent not upon its amplitude, as in the classical theory, but was constrained to be integral multiples, $n$, of a "quantum" of mode energy $E=h v$. The constant of proportionality $h$, of course, became known as Planck's constant.

In 1905 Einstein's thermodynamic analysis of the volume dependence of the entropy of an ideal Botzmann gas in the high-frequency limit of Planck's law [4], led him to form the light-quantum hypothesis and its heuristic consequence that the interaction between light and matter takes place by exchange of energy quanta. For this feat of brilliance he was awarded the Nobel prize of 1921 (which followed Planck's of 1918).

In 1909 J. J. Thomson suggested an experiment to investigate whether for extremely weak light sources the individual "units of energy" which seemed to exist in X-ray and ionisation experiments, 
distributed non-uniformly over the wavefront, would modify ordinary diffraction or interference effects. This was carried out by Taylor [25], who photographed the shadow of a needle illuminated by a gas flame through a narrow slit, and inserted smoked-glass filters successively to reduce the light intensity. He estimated the strength of his light source in the weakest case at $5 \times 10^{-6} \mathrm{ergs} / \mathrm{s}$, which he equated to the strength of a standard candle at one mile. Allowing an exposure time of about three months, Taylor created a diffraction pattern of the same form as with stronger illumination. In Thomson's opinion this indicated that the energy units could be no larger than $1.6 \times 10^{-6}$ ergs if, as he thought, more than one must be present to create an interference pattern. This is, in fact, over a thousand times greater than one of Einstein's quanta.

It is interesting to note that the idea of Einstein's quantum of light seems not to have penetrated to Cambridge even by that time. Neither Thomson nor Taylor made any reference to Einstein or Planck's work.

Later, in two papers in 1916 [26,27], and a further one in 1917 to be discusssed below ${ }^{3}$ Einstein came up with what he called himself a "magnificent light" (prächtiges Licht) on the meaning of his heuristic principle for the absorption and emission of radiation. In these papers he introduced the concepts of spontaneous and stimulated emission which led him to a new derivation of Planck's radiation law.

In 1917 in his paper entitled "On the quantum theory of radiation" [29], Einstein again made use of statistical arguments and Bohr's atomic stationary states to endow the light quantum with both momentum and energy. This was confirmed experimentally by Compton in 1923 [30]. In that same year, de Broglie [31], thinking deeply about Planck's law, proposed that matter as well as radiation should obey $E=h v$. A year later Bose [32], presented to Einstein a derivation of Planck's law using a new statistics of massless, nonconserved, spin-one particles with two polarisations (Bose-Einstein statistics). The derivation made no explicit use of electrodynamics (see below). The statistics of spin one-half particles was derived independently by Fermi and Dirac in 1926 [33,34].

Thus we see that the study of the statistics of photons was a crucial constituent in the birth of the quantum theory of both radiation and matter, but we note again, however, that in all these works the radiation field was always assumed to be in thermal equilibrium with its surrounding matter.

\section{INCOHERENT SOURCES}

In 1909 Einstein [35] had considered the problem of radiation in thermodynamic equilibrium with its surroundings. He had found that the total variance of the energy fluctuations per unit volume in a black-body cavity were given by

$$
\overline{E^{2}}-\bar{E}^{2}=h v \rho(v)+\frac{c^{3}}{8 \pi v^{2}(v)}
$$

where $\rho(v)$, is the radiative energy density given by the Planck law:

$$
\rho(v)=\frac{8 \pi h v^{3} / c^{3}}{e^{\beta h v}-1}
$$

\footnotetext{
${ }^{3}$ See Einstein and the quantum theory [28] for a full account of these historic advances.
}

where $\beta=1 / k_{B} T$, and $k_{B}$ is Boltzmann's constant. The first term is characteristic of the classical poissonian statistics of distinguishable particles and the second represents the fluctuations in energies of classical light waves. Black-body radiation was thus shown to exhibit fluctuations characteristical of both particles and waves simultaneously, the particle-like and the wave-like contributions dominating at high and low frequencies respectively.

The Boltzmann thermal equilibrium factor for the number of quanta in a mode implies that the probability of the mode containing $n$ quanta is given by

$$
p(n)=\frac{e^{-n \beta h v}}{\Sigma_{n} e^{-n \beta h v}},
$$

The first moment or mean number of quanta in the mode is, by definition, $\bar{n}=\Sigma_{n} n p(n)$, which, by summing the geometric series to infinity, gives the Planck law

$$
\bar{n}=\frac{1}{e^{\beta h v}-1} .
$$

By a simple manipulation of Eqs. (3) and (4) we see that the probability distribution of Eq. (3) has the alternative form

$$
p(n)=\frac{\bar{n}^{n}}{(1+\bar{n})^{n+1}},
$$

which is known as the "geometric" distribution. We shall see shortly that this same distribution is also a limit, for large numbers of both particles and states, of the Bose-Einstein distribution for noninteracting, indistinguishable particles placed into distinguishable particle states. Thus, in this derivation of the photon statistics of thermal or incoherent light, no concept of temperature or even radiation is actually required.

The mean square fluctuation or variance of the distribution $\overline{n^{2}}-\bar{n}^{2}$ can be calculated in a similar way to the first moment (see, for example Loudon [36]). The result is $\overline{n^{2}}+\bar{n}$. For large $\bar{n}$ this shows that the root-mean-square intensity fluctuation of an incoherent light beam is close to its average intensity. The excess fluctuation of an incoherent source is dependent both on the statistics of the fluctuations and their spectrum. Thus, for example, we very often use the model of gaussian-lorentzian light with the former term describing the statistics and the latter the spectrum. Gaussian statistics arise from some central-limit theorem operating and Lorentzian spectra from processes with a strong single relaxation time. This model fits very well, for example, the light scattering from diffusing Brownian particles in solution. Of course, to obtain the full theoretical fluctuations one must sample them for a time short compared with the inverse spectral linewidth to avoid averaging them out.

Any elementary particle can be classified by a spin quantum number which is either integral, when it obeys Bose-Einstein statistics, or half integral, when it obeys Fermi-Dirac statistics. To gain a foothold into this territory of relativistic quantum theory the reader might like to google "Balinese candle dancers" to find an easy explanation of why even a classical body does not replicate itself after one $360^{\circ}$ rotation but actually needs to go around twice.

Bose-Einstein statistics arise when a number, $r$, of indistinguishable particles with negligible interaction, are placed randomly into a number, $N$, of distinguishable particle states. There will be $\left(\begin{array}{c}N+r-1 \\ r\end{array}\right)$ possible placements, that is, the number of ways of selecting $r$ 


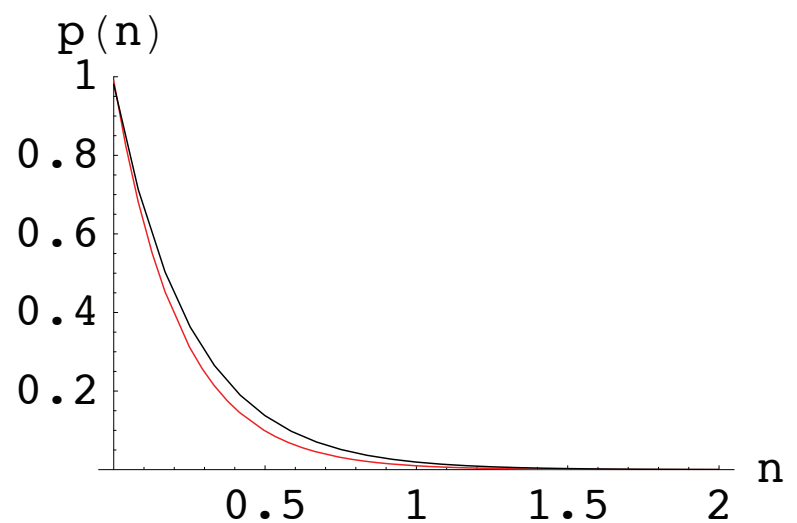

FIG. 6 Bose-Einstein distribution, $N=100,000, r=20$ (red), geometric distribution, $\bar{n}=0.02$ (black)

particles from $N+r-1$ states. These are all taken to be equally probable. The -1 comes from the fact that $N$ pigeon-hole dividers make only $N-1$ pigeon holes. (Just work out the factorial $\left(\begin{array}{l}p \\ q\end{array}\right)=p ! /[q !(p-q) !]$, for example, for 1 letter and 2 pigeon holes $)$. The probability that a given state contains exactly $n$ particles is

$$
p(n)=\left(\begin{array}{c}
N+r-n-2 \\
r-n
\end{array}\right) /\left(\begin{array}{c}
N+r-1 \\
r
\end{array}\right)
$$

that is, the normalised number of ways of selecting the other $r-n$ particles from $(N-r-1)-(n-1)$ states. In the latter expression we have necessarily removed not only the $n$ contents of the chosen pigeon hole but one of its boundaries as well.

For massless photons $r$ is not conserved and we are interested in the limit of this expression as both $N$ and $r$ tend to infinity. Remembering Sterling's formula that for large $x, \log (x !) \approx x \log (x)-x$, a few lines of Mathematica finds again the geometric distribution:

$$
p(n)=\frac{\bar{n}^{n}}{(1+\bar{n})^{n+1}}
$$

where $\bar{n}=r / n$, is the mean number of particles in each state. For light in thermal equilibrium with its source electrons, this mean value will be given by the Planck law of Eq. (4).

In Figure 6 a Bose-Einstein distribution is overlaid on a geometric distribution at sufficiently large $N$ and $r$ for it to approximate the limit of the geometric distribution. At visible light frequencies $h v / k T \approx 40$ and the geometric distribution tends to unity at low particle numbers.

This is the distribution, $p(n, T)$, which we expect to find when investigating the statistical distribution of the number of photon detections, $n$, from an incoherent light source at a given mean count rate, in a fixed sample time, $T$, much less than its inverse spectral width. Since $T$ for any conventional incoherent visible light source would be sub-nanosecond this time has been too short to achieve the full theoretical fluctuations in the pre-laser experiments and has only been achieved using a coherent laser beam and moving random scatterers, particularly from the Brownian motion of small particles. Important intensity-fluctuation experiments, however, most famously perhaps by Hanbury Brown and Twiss [37]- [44], but also earlier by Gorelik [45], Forrester, Gudmundsen and Johnson [46] and others, had already been suggested and successful measurements of the excess fluctuations of incoherent sources had been made before the advent of the laser, notwithstanding difficulties due to the short sampling times required.

We will not need to consider spin one-half particles explicitly in this paper but the distribution is even more easily derived. Viz. if, in addition to the above conditions, the Pauli exclusion principle applies so that each particle state can only be occupied by one or zero particles then Fermi-Dirac statistics ensues. In this case, all distinguishable states are equally probable with probability $1 /\left(\begin{array}{c}N \\ r\end{array}\right)$.

For comparison, the classical occupancy problem, when a number, $r$, of indistinguishable particles are placed into a number, $N$, of indistinguishable particle states when all $N^{r}$ possible placements are equally probable gives rise to Maxwell-Boltzmann statistics. In this case the probability of the partition, $\sum_{1}^{N} r_{i}=r$ is $r ! /\left(N^{r} \prod_{1}^{N} r_{i} !\right)$ and the probability $p(n)$ that a given state contains exactly $n$ particles is given by the binomial distribution

$$
p(n)=\left(\begin{array}{l}
r \\
n
\end{array}\right)(N-1)^{r-n} / N^{r}
$$

The limit of this as $N \rightarrow \infty$ is the Poisson distribution $p(n)=$ $\left(\bar{n}^{n} / n !\right) e^{-\bar{n}}$.

Note that we could replace "particle" by "ball" and "particle-state" by "bucket" and all three types of statistics would apply to classical distributions if they obey the same restrictions, for example, Feller [47], gives an example of misprints in a book which obey FermiDirac statistics. However, in spite of its historical significance, the Maxwell-Boltzmann distribution does not apply to physical particles such as photons and electrons except in the extremes of low concentration or high temperature where all three distributions converge. Otherwise, photons need to be described by Bose-Einstein statistics and electrons by Fermi-Dirac statistics.

As the sampling time, $T$, increases up to and beyond the coherence time of an incoherent source, the intensity fluctuations will be averaged out and at long sample times the integrated intensity will approach its average value. The photon-counting distribution will then be poissonian. With a model gaussian-lorentzian source of linewidth $\Gamma$ these effects can be calculated precisely although not easily [48]. In Figure 7 we show the probability distributions of intensity for various values of $\Gamma T$. The statistical properties of the light are contained in the correlations of the randomly fluctuating values of the electric field, $\mathcal{E}(r, t)$, at different points of space and time. An ideal light detector at a point in space responds not to the electric field itself but to the modulus of the square of the positive-frequency part, $\mathcal{E}^{+}(r, t)$, corresponding to annihilation of photons. The information obtainable from a single detector in this type of experiment is therefore contained in the statistical behaviour of the quantity

$$
E(T)=\int_{0}^{T} \mathcal{E}^{+}(r, t) \mathcal{E}^{-}(r, t) d t
$$

Note that this is quite different from the classical form (see Section 5 below)

$$
E_{c l}(T)=\int_{0}^{T} \mathcal{E}^{2}(r, t) d t .
$$

The feature of most importance of each distribution is its second central moment; for a gaussian-lorentzian source this is given by [48]

$$
\sigma_{T}^{2}=\frac{\langle E\rangle^{2}}{2 \gamma^{2}}\left(e^{-2 \gamma}+2 \gamma-1\right)
$$




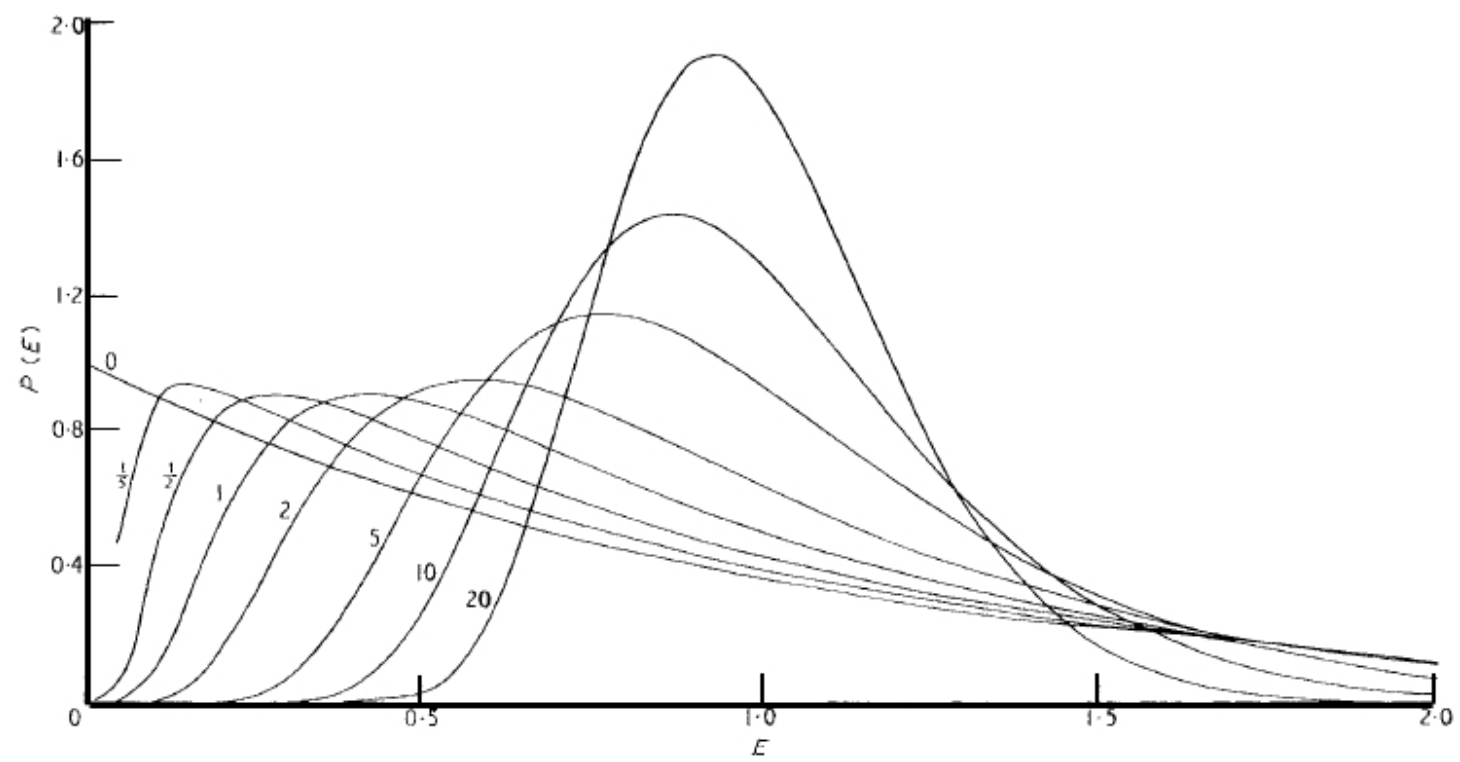

FIG. 7 The probability distributions of gaussian-Iorentzian light fields for the values of $Г T$ shown. From Jakeman and Pike, [48].

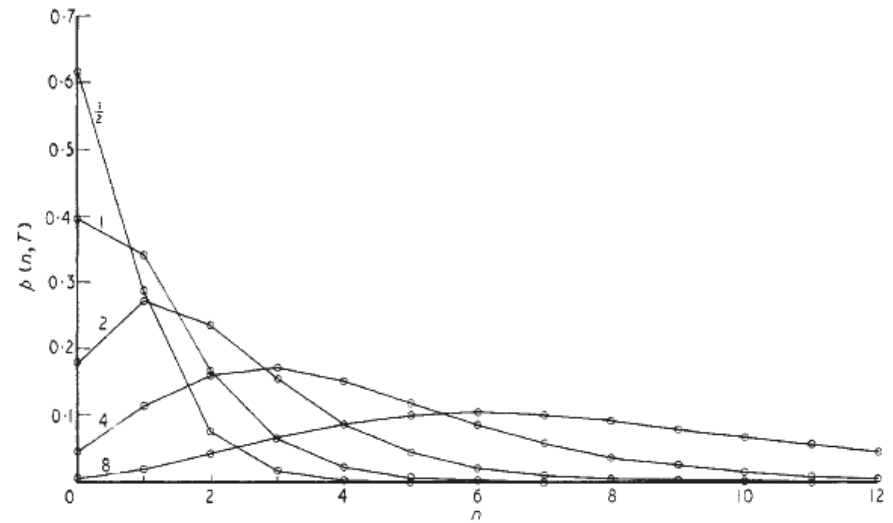

FIG. 8 Photon-counting distributions of gaussian-lorentzian light fields for $\Gamma T=5$ and the values of $\bar{n}$ shown. From Jakeman and Pike [48].

Since $P(E)$ is not directly available experimentally the photoncounting distribution [49]

$$
p(n, T)=\int_{0}^{\infty} \frac{(\alpha E)^{n}}{n !} e^{\alpha E} P(E) d E
$$

is measured, where $\alpha$ is the quantum efficiency of the detector. A quantum-mechanical derivation of this formula was given by Kelley and Kleiner [50].

The $r$ th factorial moments, $N^{(r)}$, of a random variable $n$ are defined as $\langle n(n-1)(n-2) \ldots(n-r+1)\rangle$. The normalised central moments of the intensity distribution can be shown to be equal to to the normalised factorial moments $N^{(r)} /\left(N^{(1)}\right)^{r}$ of the photoncounting distribution and these quantities, which are indepedent of quantum efficiency, are routinely used for comparison of theories with experiment. As we shall see in a moment, the expected values for an incoherent light source at short sample times are $r$ !. For a perfectly coherent source they are all unity since the $E$ of Eq. (9) is constant and thus the $P(E)$ of Eq. (12) is a delta function.

\section{THE WORK OF GLAUBER ON THE QUANTUM STATISTICS OF GENERAL LIGHT FIELDS}

It was not until after work of Townes and his colleagues published in 1954 [51], and the advent of the maser and laser that the concept of a steady light source far from thermodynamic equilibrium became a reality and of great theoretical interest.

In fact, in the first of his 1963 papers [9] Glauber states that "the quantum theory ... has had only a fraction of the influence upon optics that optics has historically had upon the quantum theory.... optical experiments to date have paid very little attention to individual photons". Indeed, Glauber's intrusion into the photon-less field of optical coherence was not welcomed by some and a running battle had to be fought for a number of years to establish that his arguments were correct. Even five years later a paper of our own [48], on intensity fluctuations of Gaussian light fields, came in for the same criticism. We were told that "there is no difference between the results obtained by classical and by quantum-mechanical calculations in this case" [52]. In our refutation, immediately following in the same journal, we had to point out yet again that an ideal optical detector measures the rate of photon detections and not the square of the electric vector. In fact, in that very paper we had contrasted the results of our quantum mechanical calculation with the quite different corresponding classical result, previously obtained by Slepian in 1958 [53]. In the barest second-quantised form, a photon annihilation operator acting on an $n$-photon state obeys $a|n\rangle=\sqrt{n}|n-1\rangle$ while a creation operator obeys $a^{\dagger}|n\rangle=|\sqrt{n+1}\rangle$, the commutator thus obeys the bosonic prescription $\left[a, a^{\dagger}\right]=1$ which has no meaning in any classical description since this would have no zero-point energy.

Many papers of this era also never questioned the assumption of gaussian stationarity, familiar in electrical engineering noise theory, for their theories of light fields. These include the work of Hanbury Brown and Twiss [37]- [44], Purcell [54], Kahn [55] and Mandel [49]. Of course, in most cases the assumption was close to the truth, However, Glauber [9] had to point out that this assumption 

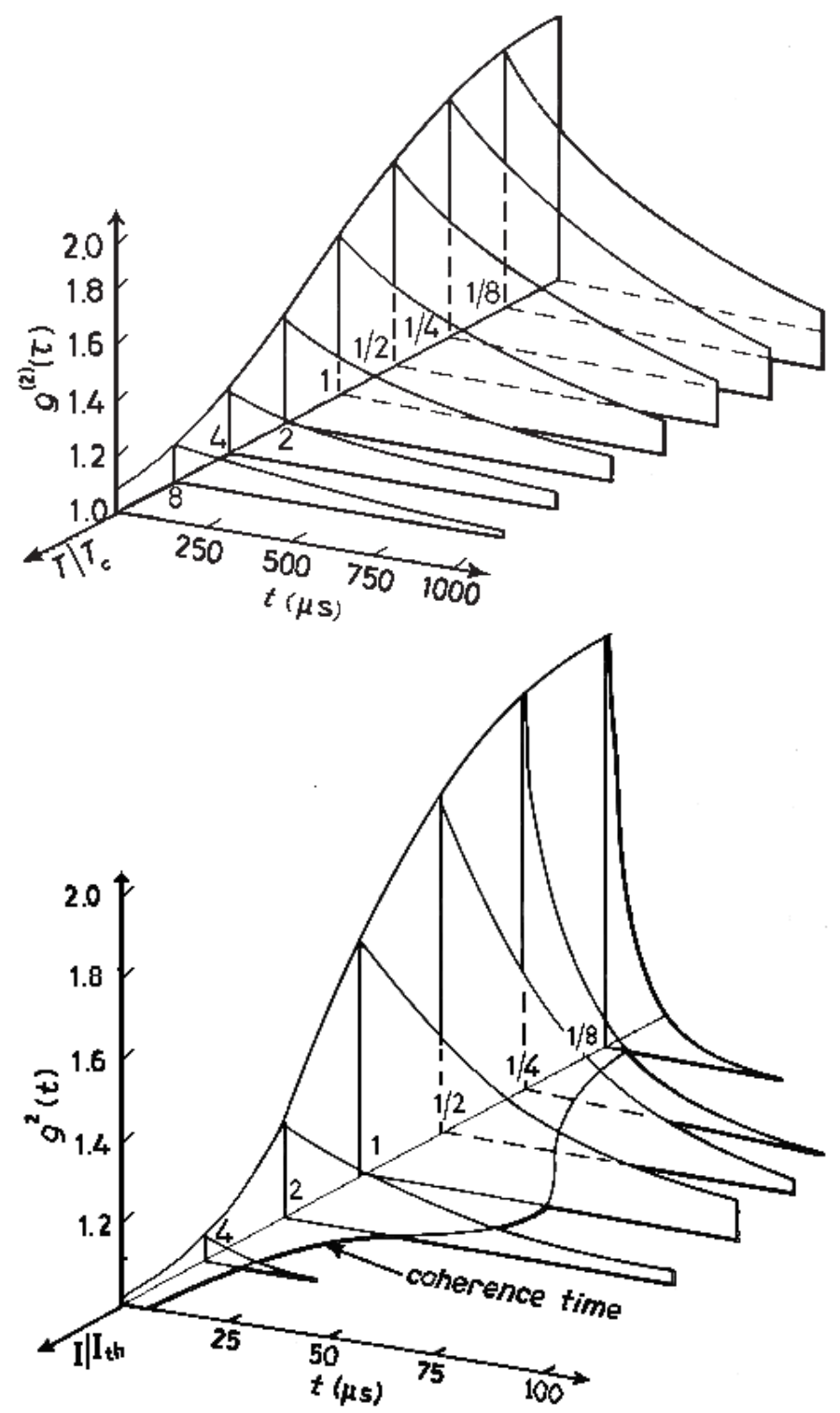

FIG. 9 Correlation and coherence for an incoherent source (left) and a laser (right).

was not generally true, an arbitrary light field needed both its statistics and is spectrum to be specified separately. To make the point clear, in Figure 9 we show the second-order correlation coefficient $g^{(2)}(\tau)$ and the coherence time (equal to the inverse of the spectral width) for, respectively, an incoherent source and a laser source about its threshold. These figures and their descriptions can be found in [56]. Glauber [9] states rather tartly but perfectly correctly that "There is ultimately no substitute for the quantum theory in describing quanta"!

In his fundamental papers on the quantum theory of optical coherence Glauber constructed a sequence of correlation functions

$$
G^{(n)}\left(\mathbf{r}_{1} t_{1} \ldots \mathbf{r}_{n} t_{n}, \mathbf{r}_{n+1} t_{n+1} \ldots \mathbf{r}_{2 n} t_{2 n}\right)
$$

for the electric-field vectors $\mathcal{E}(\mathbf{r}, t)$. For a fully coherent field, such as might be obtained from an ideal laser, he showed that the higherorder correlation functions factorize as follows :

$$
G^{(n)}\left(\mathbf{r}_{1} t_{1} \ldots \mathbf{r}_{2 n} t_{2 n}\right)=\prod_{i=1}^{n} \mathcal{E}^{*}\left(\mathbf{r}_{i}, t_{i}\right) \prod_{j=n+1}^{2 n} \mathcal{E}\left(\mathbf{r}_{j}, t_{j}\right)
$$

while for an ideally incoherent (first-order coherent Gaussian) field he gave the relations

$$
G^{(n)}\left(\mathbf{r}_{1} t_{1} \ldots \mathbf{r}_{2 n} t_{2 n}\right)=n ! \prod_{i=1}^{n} \mathcal{E}^{*}\left(\mathbf{r}_{i}, t_{i}\right) \prod_{j=n+1}^{2 n} \mathcal{E}\left(\mathbf{r}_{j}, t_{j}\right) .
$$

By photon counting with a single detector which is effectively an ideal broadband device of negligible spatial extension these reduce, for a coherent field to

$$
G^{(n)}\left(t_{1} \ldots t_{n}, t_{n} \ldots t_{1}\right)=\prod_{i=1}^{n} G^{(1)}\left(t_{j}, t_{j}\right)
$$

and for an incoherent field to

$$
G^{(n)}\left(t_{1} \ldots t_{n}, t_{n} \ldots t_{1}\right)=n ! \prod_{i=1}^{n} G^{(1)}\left(t_{j}, t_{j}\right)
$$

from which by following the arguments in Glauber's paper of 1965 [57], the normalised factorial moments of the photon-counting distributions are found to be $n^{(r)}=1$ in the coherent case and $n^{(r)}=r$ ! in the incoherent case.

\section{PUTTING ON MY OTHER HAT}

Armed with a comprehensive new quantum theory of photon counting and optical coherence from the work of our study group I soon became eager to get into the laboratory again to measure the statistics of optical light fields but, with my background of photon counting of $\mathrm{X}$-ray photons, I soon became puzzled as to why there seemed to be no literature on the counting of photons at frequencies in the visible range. After all $h v$, although not as large as for X-rays, would still be something like forty times room temperature.

Our biggest manufacturer of photomultiplier tubes in the UK was EMI and on my first visit to them I was met with bafflement when I asked about photon-counting efficiencies. They assured me that what their photomultipliers measured was lumens not photons. Actually, they were almost right since after detailed testing in my laboratory it turned out there was no single-photon peak to be seen in the output of any of their offerings. With a constant light input the pulse count kept increasing with overall voltage no matter how high. The products of all other manufacturers we tried behaved in the same way. To be fair to EMI they did listen and later went on to produce a range of excellent phototubes with their own private venture funds.

When a photoelectron is emitted from a photocathode it gets mutiplied by secondary emission at each dynode in the structure, see Figure 5. At each stage the increasing current can ionise any residual gas in the tube and the positive ions created will race back down the tube towards the cathode, in turn causing further "afterpulses" a few microseconds later, the same process can then repeat many times. Additional noise sources could arise from the high voltages at the base of the tube and in the following circuitry. These processes at first blighted all our efforts to make a respectable, theoretically well behaved, single-photon counter. A number of workers by-passed the problem at the time by integrating regardless the total current or counts over some threshold over a fixed period [58]- [63]. These and other pioneering early results are reviewed in a comprehensive summary by Lax and Zwanziger [64].

Of course, at a fixed voltage the output current would increase with light input so unless one tried to digitise the count rate, which should, 
of course, saturate when essentially all the photoelectrons from the cathode were amplified above the discriminator threshold, the problem went unnoticed, but the calibration in lumens would have had to be carried out with a specified value of electron multiplication.

Eventually we had the good fortune to get hold of a tube developed by E. H. Eberhardt, at ITT, Fort Worth, Indiana [65, 66, 67]. This was called a "star tracker" and the secret of its success was that only a small "active" area of the photocathode was electrostatically focussed onto the first dynode. Photoelecton emission from anywhere else on the cathode was blocked by a pinhole in a metal shield at the focus. All our successful early work used these tubes.

A feature of nuclear counting had always been the need to allow for the unavoidable "dead time" following the registration of each count. Accounts of these procedures, which are necessary for accurate comparisons with theoretical photon-counting distributions, and the use of phototubes for photon counting can be found in $[68,69]$.

\section{A SIX-DECADE LEAP IN RESOLUTION}

In conventional optical spectroscopy the more closely spaced are spectral lines the more difficult it becomes to resolve them. The most elaborate interferometric spectrometers in existence, one of which we built ourselves, a Fabry-Perot interferometer, see [70], finally give up at line spacings of about $1 \mathrm{MHz}$. It is a curious accidental fact that the linewidths of pre-laser light sources were never narrow enough to warrant higher resolution than could be achieved by interferometry. The sharpest low-temperature mercury vapour isotope lamp line, for example, has a width of about $100 \mathrm{MHz}$. Only the advent of the highly monochromatic laser raised the demand for higher resolution. The measurement of the correlation of photons, on the other hand, becomes easier as the spectral line separation or spectral linewidth gets smaller, since photon bunching arises from beat frequencies of the quantum wave field which become slower with reduced separation and thus more convenient for electronic circuitry. This, in itself, would allow an extension of spectroscopic resolution down to the intrinsic linewidths of laser sources themselves. However, although the theoretical phase-diffusion time of a laser can be in the region of $1 \mathrm{~Hz}$, in a normal laboratory acoustic environment practical linewidths are at least $10 \mathrm{KHz}$ and more often are around $1 \mathrm{MHz}$ or broader. An elaborate interferometer would thus seem still to be necessary for most problems.

That this is not so is due to a further powerful feature of the photoncorrelation method, namely, that the optical carrier frequency is not measured. The theory of photodetection shows that it is the wavefield envelope which gives the photon-detection probability which is, therefore, dependent only on difference frequencies in the optical signal. Phase noise in the carrier cancels out in the detection process as long as it is slow enough compared with the phenomena under study or, in a scattering experiment, as long as large path differences are not present. The photon-correlation function is thus independent of the source linewidth for a wide range of sources, which includes most lasers. Spectroscopy down to $1 \mathrm{~Hz}$ is therefore feasible, extending previous techniques by some six decades in one fell swoop.

A very bright non-laser source can sometimes be used as a probe for photon-correlation studies. but this is not generally a practical proposition. We have thus another curious accidental fact that, even

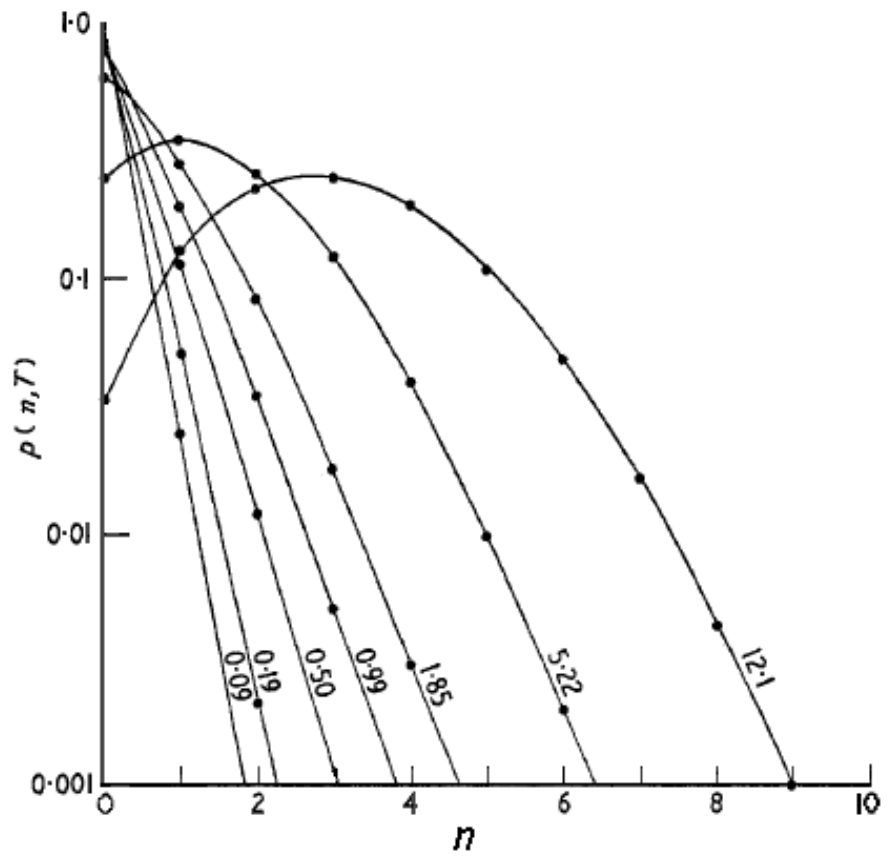

FIG. 10 Photon-counting distributions of a single-mode laser through threshold. The curves are labelled by the ratio of the intensity to that at threshold. The sampling time was $3 \mu$ s with negligible temporal integration of the fluctuations for all the ratios shown. (Reproduced from [74]).

had they been available, photon-correlation methods would not have been so practical to apply in pre-laser days.

\section{THE STATISTICS OF LASER LIGHT}

In Section 5 we saw that the normalised factorial moments of the photon-counting distribution for ideal coherent light were all unity, as pointed out by Glauber, and elaborated elegantly in his exposition of the overcomplete set of coherent basis states for the light field referred to above.

These simple values for normalised factorial moments follows from Poisson statistics for the photon-counting distribution. Such states are produced by a laser operating well above its threshold. At lower pump rates the output will change to that of an incoherent gas discharge. The transition from one to the other became of interest both theoretically and experimentally. A suitable theoretical model for these studies of gas lasers was published in 1967 by Scully and Lamb [71].

In the years following there have been many attacks on the theory of the laser, which is a completely non-trivial problem in quantum optics, see, for instance, Pike and Sarkar, The Quantum Theory of Radiation [72]. Some ten years ago, in the context of the proposition of thresholdless lasing, we returned to the problem and for the first time were able to solve the three-level model, in the form of an analyic recursion relation for the photon-counting distribution to all orders in the coupling constant between the cavity modes and the lasing atoms, in both two- and three-level cases [73].

In Figure 10 we show a set of experimental results for the photoncounting distributions of a single-mode laser taken from an order of magnitude below to an order of magnitude above its intensity at 

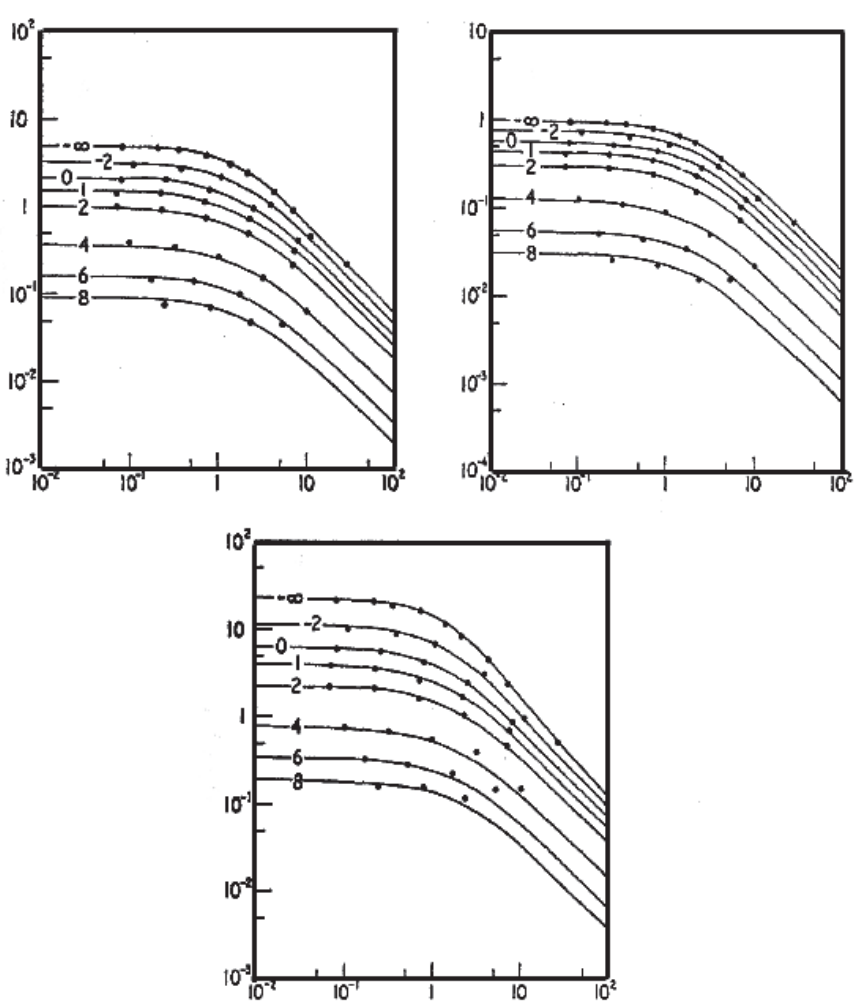

FIG. 11 Second, top left, third, top right and fourth, bottom, normalised factorial moments for the photon-counting distributions of Figure 10, horizontal axes are the ratio of sample time to the effective coherence time and the curves are labelled with the pump parameter. (Reproduced from [74]).

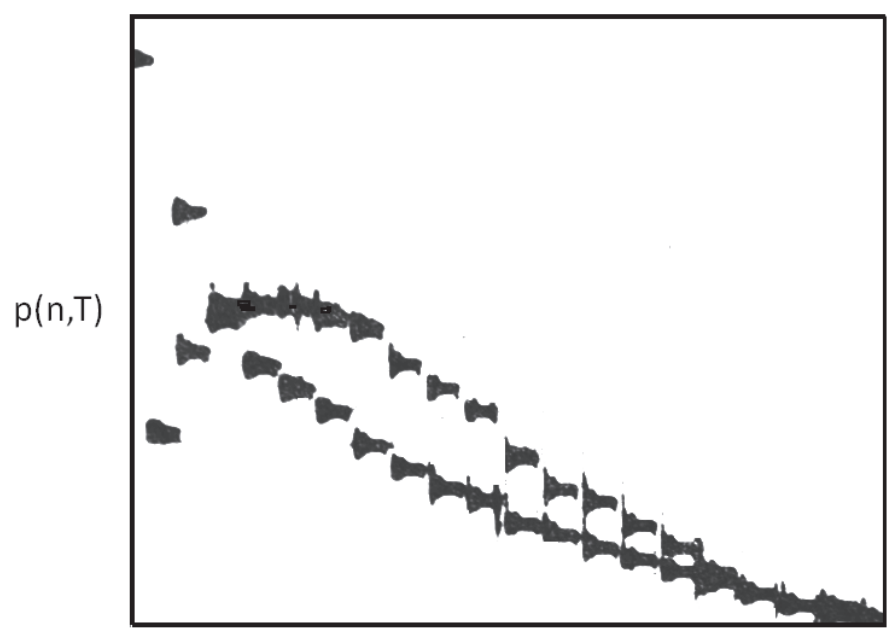

$\mathrm{n}$

FIG. 12 Coherent and incoherent photon-counting distributions.

threshold. In Figure 11 theoretical predictions of the second, third and fourth normalised factorial moments from the experimental results of Figure 10, are compared with theoretical predictions by Lax and Zwanziger in [75].

As an anecdote, Figure 12 shows photon-counting distributions for the two extremes of incoherent and coherent light on the same screen. We took this experiment to the French Physical Society Exhibition in Paris in 1966. It actually mimics a figure from Bruhat's book on thermodynamics. Professor Kastler came by our stand and immediately exclaimed "Ah, la loi des bosons!". Bruhat had brought Kastler to the Ecole Normale and he was clearly familiar with the im- plications of our work. He was later to use the figure in his revision of Bruhat's text. A happy result was that he and Professor Bernardini kindly invited me to give a plenary lecture at the inaugural meeting of the Europan Physical Society in Florence in 1969.

\section{ZERO CROSSINGS, CLIPPING AND OPTICAL RADAR}

To a radar establishment the laser could be regarded as just another coherent radar transmitter, albeit some five decades higher in frequency than those previously available. This five decades difference, exploited in an optical radar system, would reduce diffraction spread and hence increase pointing accuracy by the same linear factor and would increase Doppler sensitivity to target velocity by the same amount. The study and design of optical radars was therefore of interest, and it was in considering the sensitivity of optical detection that it was realised that the fundamental limitations on performance would be quite different from those at microwave frequencies. In the latter case it is thermal (Johnson) noise energy which. in the end, limits the sensitivity of a system, whereas the optical photon energy is greater than $k T$ by a factor of 40 or so and should therefore dominate any thermal currents present.

The design of coherent optical systems was thus unfortunately (or perhaps fortunately from our point of view) not just a question of scaling the formulae of microwave radar by the appropriate wavelength factor. A new set of foundations based on the quantum theory of light had to be laid. In principle, each tiny quantum of light could be detected and processed individually. One photon per second of flux corresponds roughly to the light which an observer in London would see from a candle at the distance of New York.

Temporal correlation of photon events were eventually conceived with the aim of creating a novel type of ultrahigh-resolution spectroscopy. This exploited the variation of the statistical properties of the photon stream with optical linewidth which had been postulated and verified earlier. In essence, the photons arrive bunched with a finite characteristic time related to the inverse of the optical linewidth, as opposed to exhibiting random arrivals as with a perfect laser. Correlation compares the photon arrivals with those at earlier times by multiplication and averaging to extract periodicities or decay times present in the signal. To overlap the region of conventional optical spectroscopy, very high-speed multiplication of photon numbers was desired (each calculation should be completed in the order of $100 \mathrm{~ns})$.

This was not, in fact, at all possible at the time, but by adapting a radar technique of one-bit quantisation to our purpose, which reduced the multiplications to simple gating operations we achieved our aim. In the radar form this technique made use of a theorem of Van Vleck and Middleton dating from the second world war which showed that the spectral content of a random signal lay in the positions of its zero crossings. Dr Jakeman and I found a practical one-bit analogue for a sampled photon train which replaced the crossing of zero voltage by the crossing of a preset integral photon number [76], this was the patented secret at the heart of our early instruments. I remember feeling some pride on a visit to the Gordon McKay Lab. at Harvard in the early 70's to see our formula

$$
\frac{\left\langle n_{k}(0) n(\tau)\right\rangle}{\left\langle n_{k}\right\rangle\langle n\rangle}=1+\frac{1+k}{1+\langle n\rangle}\left|g^{(1)}(\tau)\right|^{2}
$$




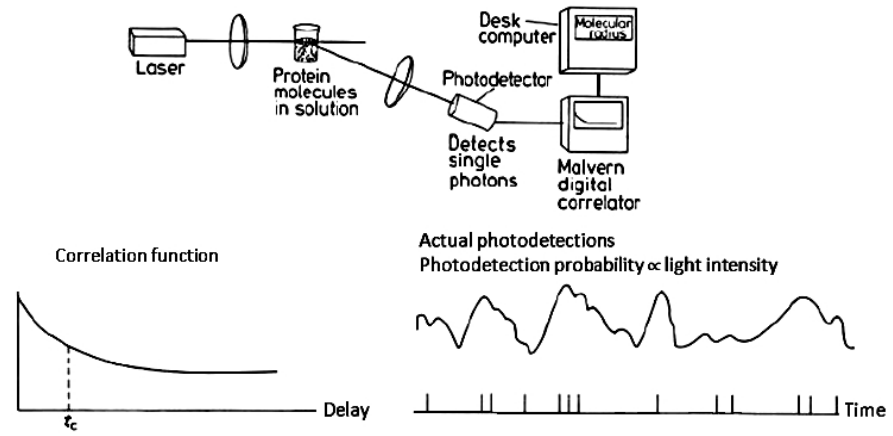

FIG. 13 Photon-correlation spectroscopy for particle sizing.

pinned up on the wall. $k$ is the clipping level, so little information is lost at any count rate if $k$ is chosen near to the mean and, of course, the multiplications by one bit can be done at lightning speed, rendering the whole thing possible.

\section{PARTICLE SIZING}

The experimental arrangement for particle sizing is shown in Figure 13. One of our first instruments was acquired by Arthur Peacock at the Nuffield Department of Clinical Biochemistry, University of Oxford and the fiftieth anniversary year of the laser happens also to be the fortieth anniversary year of our first paper to Nature demonstrating the use of photon-correlation spectroscopy to measure the hydrodynamic radius of biological molecules. We were also encouraged in this direction by the interest of Walter Gratzer of King's College London. With Peacock's group, we investigated the oxygen carrier, haemocyanin, from the whelk, murus trunculus, collected off the shores of Malta, and also measured the common molecules bovine serum albumin and lysozyme as controls. The apparatus measures the linear diffusion coefficient, which is converted to hydrodynamic radius using the Stokes-Einstein equation. Radii of such molecules and chemical salts, in suitable buffer solutions and concentrations, are in the 5-20 nm range; nonotechnology, in fact, long before it became a household word. The great advantage of the photon correlation spectroscopy method was that the measurements could be taken of low molecular weight molecules in low concentrations in short times. Our paper was published on July 18th 1970 [77]. Our ad hoc technique for generating an incoherent light source for testing photon statistics, as recounted in the introduction, had developed beyond anything which we might have imagined.

In fact, another of the things it led to arose from the obstinate refusal of data processing algorithms of any breed or recipe to find the particle-size distribution from the apparently highly accurate digital correlation functions obtained by correlating tens of millions of photons. Although the mean size could be and was routinely used, the actual distribution was not. The inversion requires a numerical inverse Laplace transform. This problem, although heavily trodden for many years, still needed more work. With John McWhirter at Malvern our first contribution was a new approach to the finite inverse Laplace transform in 1978 [78]. The mystery, although not exactly solved, became understood in the context of a burgeoning field known as "Inverse Problems", into which I have since transferred a lot of allegiance and have built up a parallel exciting circle of new friends and colleagues.

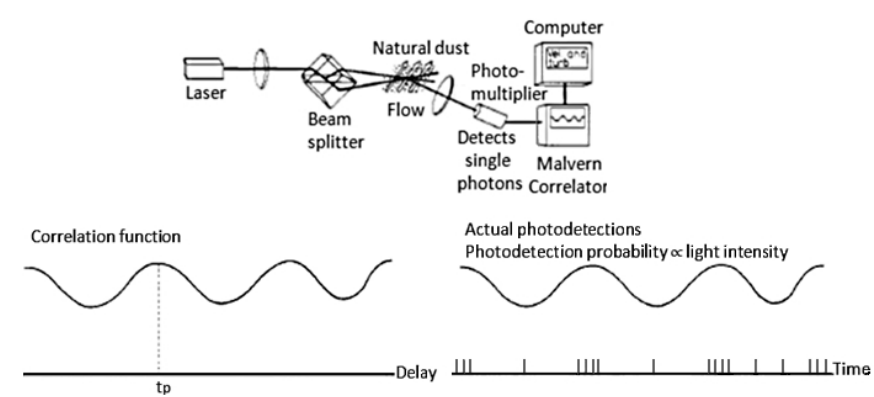

FIG. 14 Photon-correlation spectroscopy for velocimetry.
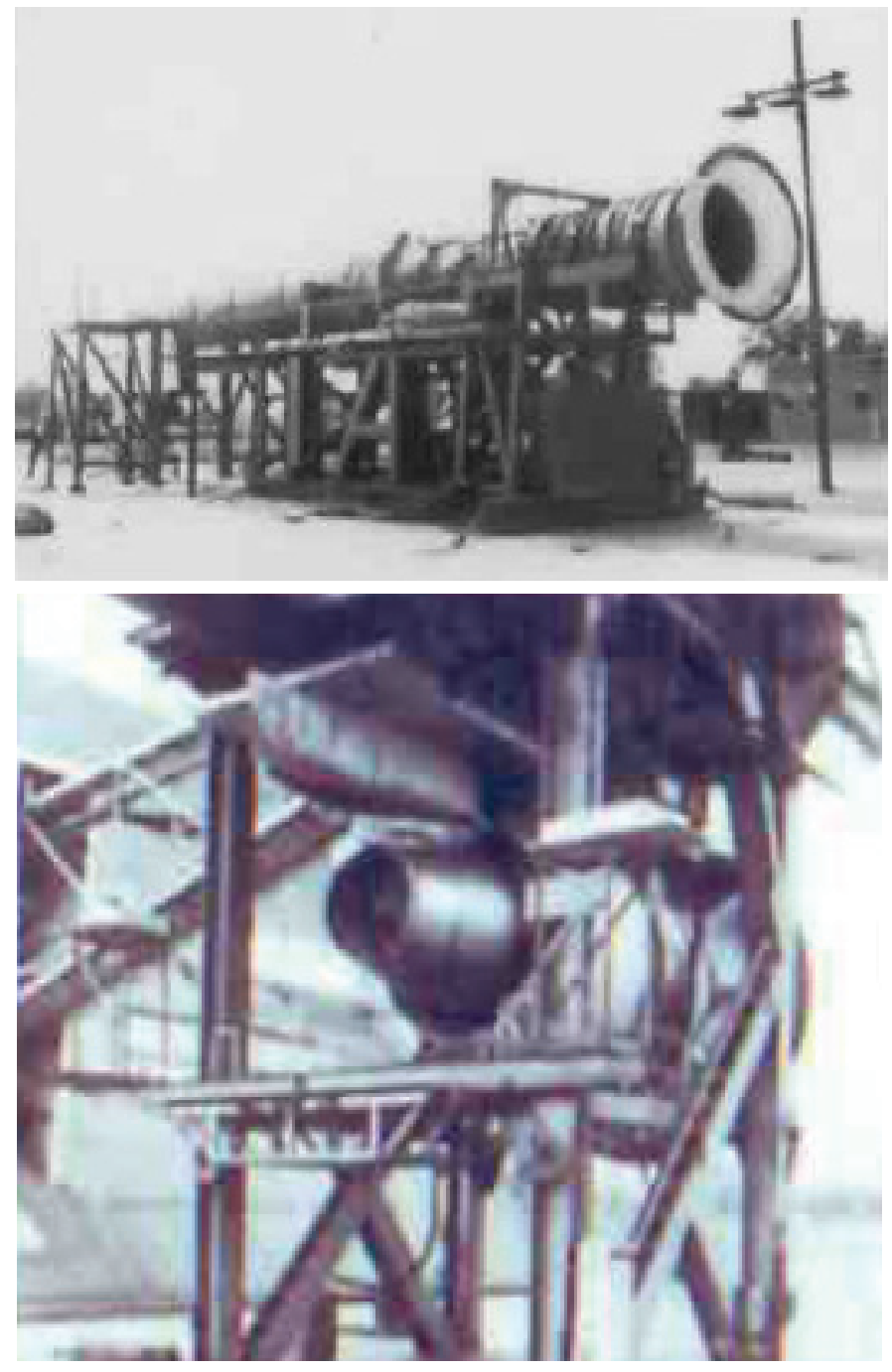

FIG. 15 Studies of the Olympus 593 (Concorde) and RB211 aeroengines at the Royal Aircraft Establishment.

\section{AERONAUTICAL ENGINEERING}

An experimental arrangement for velocimetry is shown in Figure 14. Some examples of aeronautical applications are shown in Figures 15-18. Small "seed" particles, either naturally present or introduced artificially into an air flow, scatter a laser beam with a Doppler shift proportional to velocity. By optical mixing at the detector this shift is measured as the period of an oscillating photoncorrelation function. In closer-range applications two beams are projected and the difference of the Doppler shifts is measured. This arrangement can also be understood by considering the two incident beams to form interference fringes which the particles pass through. 

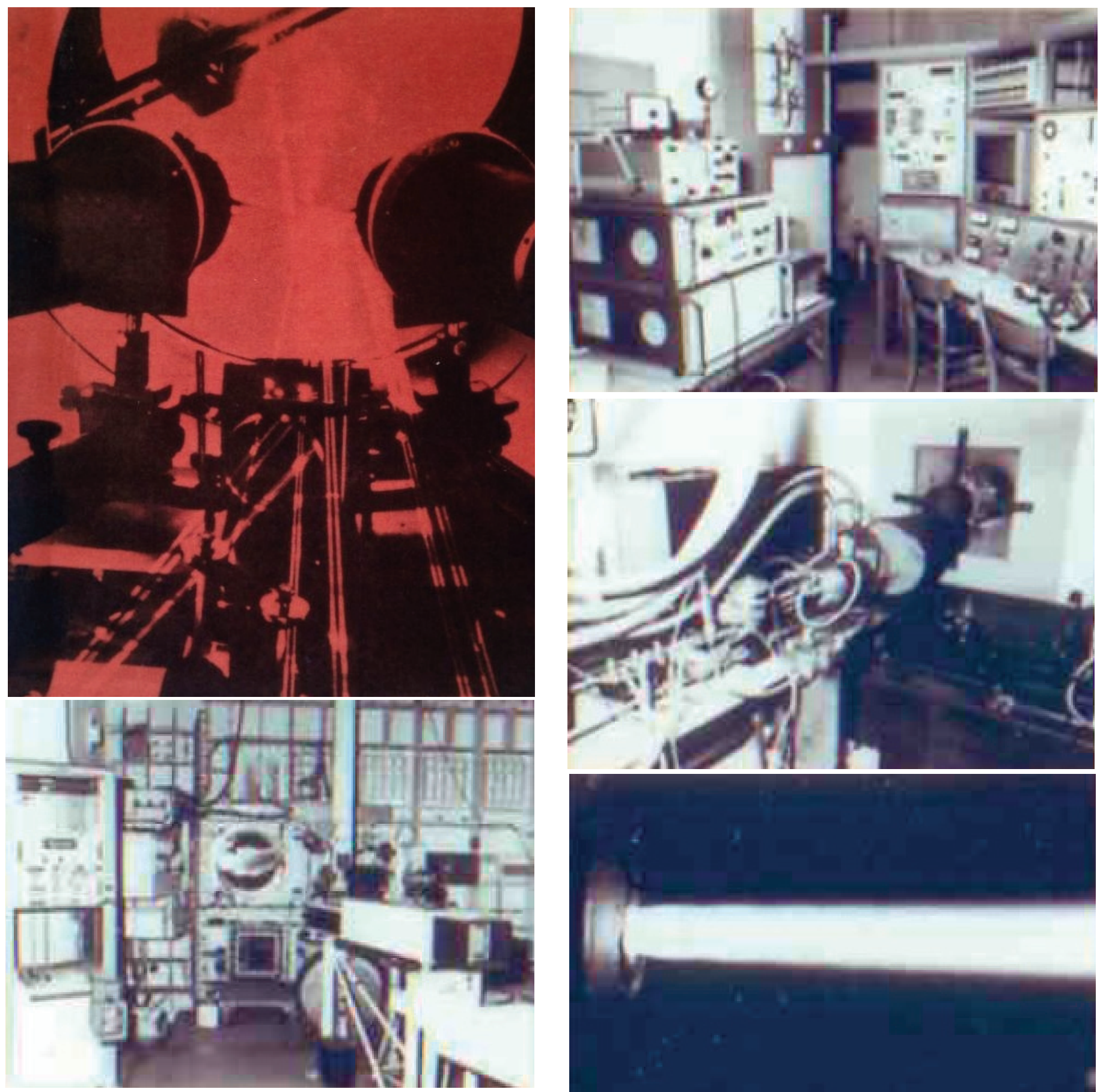

FIG. 16 Studies of aerodynamic flow fields in wind tunnels at the Royal Aircraft Establishment.
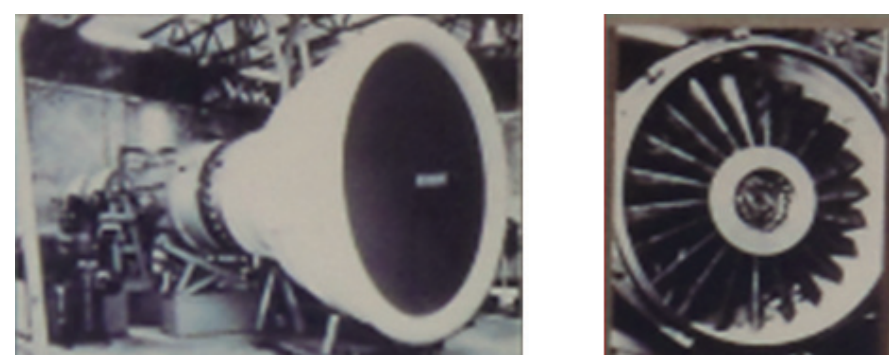

FIG. 17 Studies of an aeroengine at the General Electric Company.

The Doppler-difference frequency is the same as the fringe-crossing frequency.

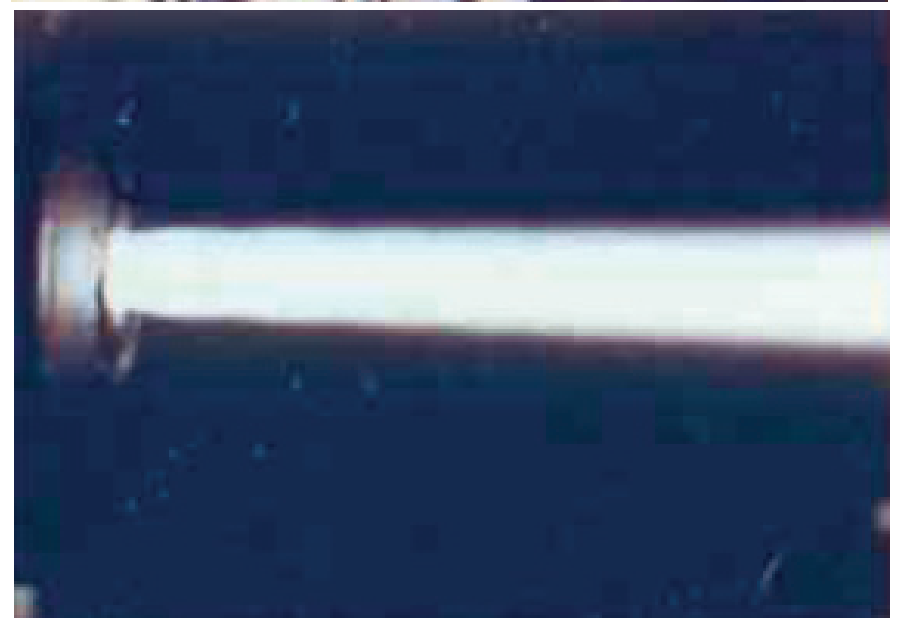

FIG. 18 A study of a liquid-fuel rocket engine at the Fraunhofer Institut für Chimie der Treib und Explosivstoffe.

In addition to the Queen's Awards to the company which I have mentioned, happily we have had a number of other awards for our work, among which perhaps the most splendid was the UK's premier award for engineering, the MacRobert Award. In Figure 19 you may see a photograph of the awardees (identified in my acknowledgements below) after receiving the medals and prize at Buckingham palace in 1977. Just as proud were we to receive congratulations at the time from many friends and colleagues around the world. Particularly appreciated were beautiful cards from Charles Townes, who will be with us this year in England for our own 50th anniversary of the laser celebrations and Alfred Kastler, who unfortunately is no longer with us but who was always a good friend and most supportive of our 


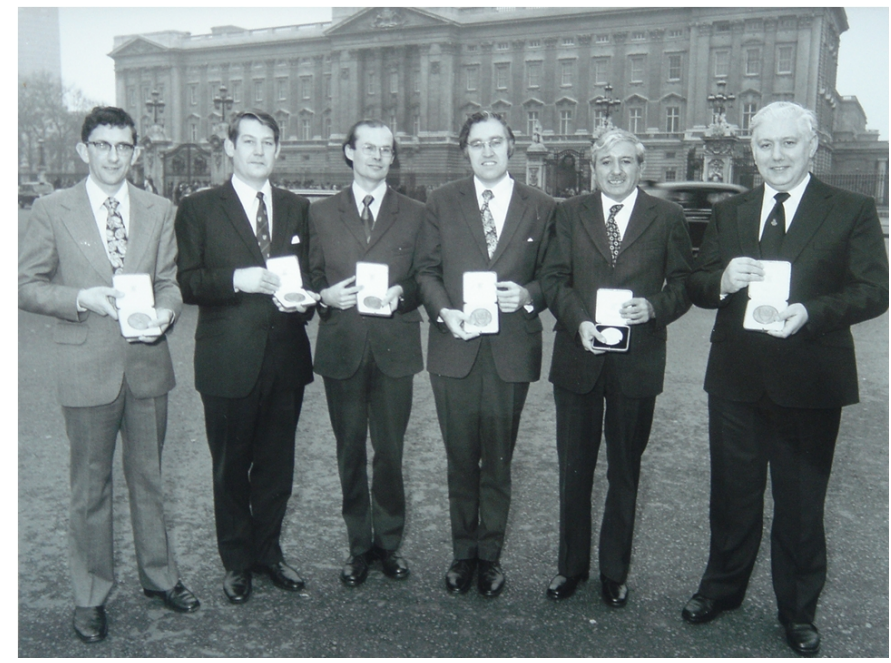

FIG. 19 The Macrobert Award, Buckingham Palace, 1977. From left, E. Jakeman, R, Jones, C. Oliver, R. Pike, R. Lees, T. Trudgill.

work. In his memory I will reproduce in Figure 20 his lovely card of 1978.

\section{ACKNOWLEDGEMENTS}

I will single out first for thanks and acknowlegement my coworkers at Malvern in the very early days, namely Alistair Johnson, Tom Mclean, Eric Jakeman, Chris Oliver and Robin Jones, also Steve Trudgill the then head of Malvern Instruments, who took a big commercial plunge into the unknown which fortunately paid off, and Roland Lees, the director of the Royal Radar Establishment who gave us his full support for this esoteric research for a defence establishment. A little later at Malvern we were joined by Michael Vaughan returning from a Fellowship at Princeton, Peter Pusey coming back from the Watson Lab at Yorktown Heights and Rodney Loudon from UCB. All three played a huge part in our research group for a number of years. Eric had done a spell at UCLA and I at MIT so it seems that we should all acknowledge valuable further education in the USA. At Malvern Instruments we owe a debt of gratitude to our colleagues who were there from the beginning, Bill Woodley, a life-long friend, who after the initial developments at the company in Malvern, handled the scene in France for many years, and similarly Maurice Wedd, who now leads UK delegations on international standards on particle characteristation for the Royal Society of Chemistry. Robert Brown joined us later in Malvern and made significant contributions, particularly when fibre optics came along and also Stuart Swain who was a very effective theorist in quantum statistics. Amongst visiting researchers there were Norman Ford who helped in the design of the goniometer of Figure 3 and Judy Brown from Wellesley and the MIT media Lab., then Danny Walls and Klaus Schätzel (both deceased at an unkind early age, but never forgotten). Beyond Malvern, John Abbiss at RAE, Farnborough, Anthony Smart at Rolls Royce, Derby, Robin Elder at Cranwell, Phil Bourke at the Whittle Labs in Cambridge, Tony Birch and Roger Brown at the Gas Board in Birmingham were all members of our lively UK correlator club which played a key role in our progress at the time. Overseas, I will add Mario Bertolotti in Rome, Nicole Ostrowsky in Nice, Ugo Palma in Palermo, Salvatore Solimeno in Naples, Herman Cummins at NYU, George Benedek at MIT, Roy Glauber at Harvard, Eli Yablonovitz at UCB/UCLA and Bob Hell-

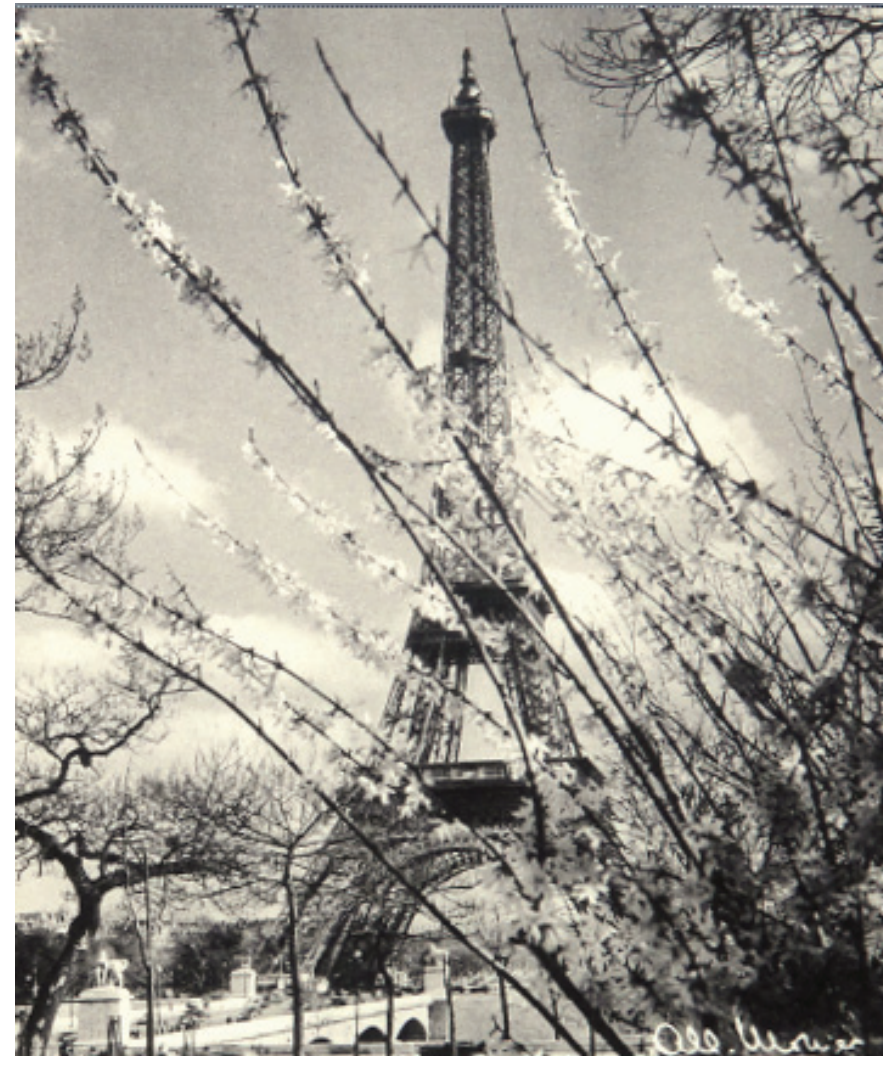

$$
\text { January 11, } 1978
$$

21 - PARTS

Lo Tour Eiflel $x \in$ an $x^{2}$ fike,

$$
\text { Owes very lafey to bearn that }
$$$$
\text { You and jou teran have been }
$$

$$
\text { divhngristed of the }
$$

Mar Rober awad.

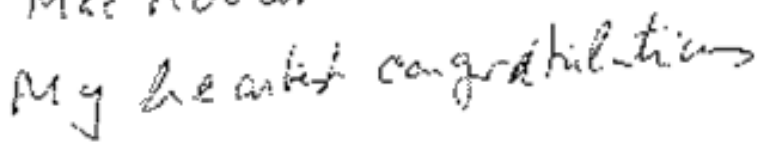

$$
\text { Les Photographies Albert Monier }
$$

Repracuction reseryee

Ciliugion C.R.P., ZI Soulevard Aroga-9i Chlly Mazarin

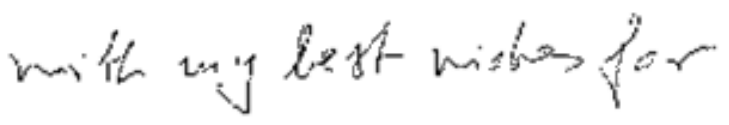

$$
\begin{aligned}
& \text { Whe new } \frac{4}{1} e \mathrm{CH}
\end{aligned}
$$

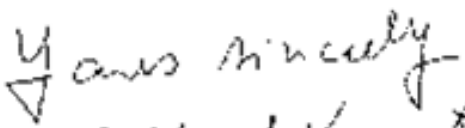

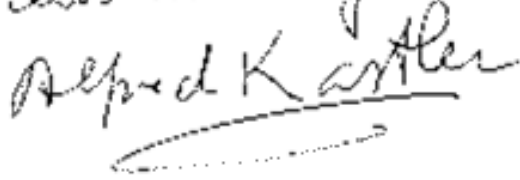

FIG. 20 Card from Prof Kastler in 1978.

warth at USC all for inspirational friendship and support in Quantum Optics from a long way back. Lars Danielsson and Ulf Sjödin de- 
serve a grateful mention for joint experiments in rocket engines and capilliary blood flow, respectively, during a stimulating "Swedish period" with reciprocal visits for a number of years. Let me also add the many luminaries listed below who willingly joined my editorial board when I decided to start up and edit the journal "Quantum Optics" from King's College London in 1989 with the encouragement of Seweryn Chomet, a physicist in the Department (and also polymath, author, journalist, historian, and publisher according to his wikipedia entry). This was first published jointly by the Institute of Physics, UK and Newman-Hemisphere, UK, and later taken on by the Institute of Physics. Today it has become merged into their Journal of Physics B. We claimed this to be the first journal devoted entirely to the field of quantum optics and, as can be seen, (present company excepted, of course) its first board of editors makes a roll call of achievers in the field worthy of recording for this historical anniversary.

To pay acknowledgements on the data-processing, inverse-problems side of laser scattering, which has spread to many other disciplines, I have to thank particularly Geoff de Villiers at Malvern, Mario Bertero of the University of Genoa and Pierre Sabatier of the University of Montpellier for setting me up and sustaining me in what has turned virtually into a second career. I would also like to mention a continuing fruitful collaboration with Tuncay University of Texas at Arlington in inverse problems in speech acoustics (sound rather than light scattering but with theoretical overlap). In this field I similarly have to thank the first editorial board members of the Journal "Inverse Problems", which I initiated when V. P. for publications at the Institute of Physics, particularly Pierre Sabatier, who graciously agreed to take on the onerous task of acting as its first editor and helped set it up as a leading journal in its field where it stands today; the Institute of Physics Publishing Division has done a magnificent job on this Journal as well. Pierre and I also collaborated on a mammoth millenium two-volume book set with 100 contributed articles on scattering in its many aspects in physics [79], for which we warmly thank all the authors.

I have to stop there since, in truth, there are far too many entangled scientific and commercial webs of friends and colleagues worldwide, grown up over the period which this paper describes, to attempt any more; my sincere thanks go to them all; I hope if they get to reading this they will know who they are.

Finally, I have to say also that, where relevant, I have leaned heavily in this laser anniversary contribution on descriptions which I have published previously along the way and, in particular from an article specifically on the Malvern Correlator of 1979 [80].

Quantum Opt, 1, (1989), Editorial Board As listed in the Journal:

- Hon. Editor: ERP, King's College, London, UK,

- Deputy Editor: M Teich, Columbia University, New York,

- G S Agarwal, University of Hyderabad, India,

- H J Carmichael, University of Oregon, USA,

- P D Drummond, University of Auckland, New Zealand,

- E Giacobino, Université Pierre et Marie Curie, Paris, France,

- R Glauber, Harvard University, USA,
- P Grangier, Institut d'Optique, Orsay, France,

- F Haake, Universität Gesampthochschule Essen, FRG,

- H A Haus, Massachusetts Institute of Technology, USA,

- R W Hellwarth, University of Southern California, USA,

- E Jakeman, Royal Signals and Radar Establishment, Malvern, UK,

- G Khitrova, University of Arizona, USA,

- L A Lugiato, Politecnico di Torino, Italy,

- L Mandel, University of Rochester, USA,

- A Miller, University of Central Florida, USA,

- B E A Saleh, University of Wisconsin, USA,

- S Sarkar, Royal Signals and Radar Establishment, Malvern, UK,

- K Schätzel, Institut für Angewandte Physik der Universität Kiel, FRG,

- R E Slusher, A T \& T Bell Laboratories, Murray Hill, USA,

- S Swain, Queen's University of Belfast, UK,

- A R Tanguay, University of Southern California, USA,

- P Tombesi, Università degli Studi di Roma, La Sapienza, Italy,

- D F Walls, University of Auckland, New Zealand,

- H Walther, Max-Planck-Institut für Quantenoptik, Garching, FRG,

- H Welling, Institut für Technische Optik der Universität, Hannover, FRG,

- B Wilhelmi, Akademie der Wissenschaften der DDR, Berlin, GDR,

- Y Yamamoto, Nippon Telegraph \& Telephone Corporation, Tokyo, Japan,

- H P Yuen, Northwestern University, Evanston, Illinois, USA.

\section{References}

[1] M. Planck, "On an Improvement of Wien's Equation for the Spectrum" Verh. Deut. Phys. Ges. 2, 202-204 (1900).

[2] M. Planck, "On the Theory of the Energy Distribution Law of the Normal. Spectrum" Verh. Deut. Phys. Ges. 2, 237-245 (1900).

[3] M. Planck, "Letter to R W Wood - 7th Oct 1931" in The Archives of the Center for the History of the Philosophy of Physics at the American Institute of Physics (New York City, USA, 1931).

[4] A. Einstein, "Concerning an Heuristic Point of View Toward the Emission and Transformation of Light" Ann. Phys.-Leipzig 17, 132148 (1905).

[5] R. Ladenberg, "Research on the Anomalous Dispersion of Gases" Phys. Z. 48, 15-25 (1928). 
[6] V. A. Fabrikant, PhD thesis (1940), quoted in F. A. Butayeva, and V. A. Fabrikant, Investigations in Experimental and Theoretical Physics, A Memorial to S. G. Landsberg, 62-70 (U.S.S.R. Academy of Science Publications, Moscow, 1958).

[7] M. Bertolotti, The History of the Laser (Institute of Physics Publishing, Bristol, 2005).

[8] J. Brossel, and A. Kastler, "La détéction de la résonance magnétique des niveaux excités - l'effet de depolarization des radiations de résonance optique et de fluorescence" C. R. Acad. Sci. (Paris) 229, 1213-1215 (1949).

[9] R. J. Glauber, “Photon Correlations" Phys. Rev. Lett. 10, 84-86 (1963).

[10] R. J. Glauber, "The Quantum Theory of Optical Coherence" Phys. Rev. 130, 2529-2539 (1963).

[11] R. J. Glauber, "Coherent and Incoherent States of the Radiation Field" Phys. Rev. 131, 2766-2788 (1963).

[12] T. A. Longo, I. Feinberg, and R. Bohn, Universal High-Level Logic Monolithic Circuits 9, 66 (Electron Devices Meeting, 1963 International, 1963).

[13] J. L. Buie, "Coupling Transistor Logic and other Circuits" U. S. Patent 3,283,170 (1966).

[14] T. P. McLean, and E. R. Pike, "The Photon-Counting Distribution for Gaussian Light" Phys. Lett. 15, 318-320 (1965).

[15] F. A. Johnson, T. P. McLean, and E. R. Pike, "Photon-Counting Statistics" in Proceedings of the International Conference on the Physics of Quantum Electronics 715 (Puerto Rico 1965)

[16] E. R. Pike, “Light and Movement, Using Coherent Light to Measure Particle Velocity" Phys. Bull. 109-112 (1976).

[17] H. Z. Cummins, and E. R. Pike, (eds.), Photon-Correlation and Light-Beating Spectroscopy (Plenum Press, London, 1974).

[18] H. Z. Cummins, and E. R. Pike, (eds.), Photon-Correlation Spectroscopy and Velocimetry (Plenum Press, London, 1977).

[19] P. Tombesi, and E. R. Pike, (eds.), Squeezed and non-classical light 190, (Plenum Press, London, 1989).

[20] E. R. Pike, and J. B. Abbiss, (eds.), Light Scattering and PhotonCorrelation Spectroscopy 40, (Kluwer, Dordrecht, London, 1997).

[21] Y. Yeh, and H. Z. Cummins, "Localized Fluid Flow Measurements with an He-Ne Laser Spectrometer" Appl. Phys. Lett. 4, 176-178 (1964).

[22] N. C. Ford, Jr. and G. B. Benedek, "Observation of the Spectrum of Light Scattered from a Pure Fluid Near Its Critical Point" Phys. Rev. Lett. 15, 649-653 (1965).

[23] J. B. Lastovka, and G. B. Benedek, "Spectrum of Light Scattered Quasielastically from a Normal Liquid" Phys. Rev. Lett. 17, 10391042 (1966).

[24] S. B. Dubin, J. H. Lunacek, and G. B. Benedek, "Observation of the Spectrum of Light Scattered by Solutions of Biological Macromolecules" P. Natl. Acad. Sci. USA 57, 1164-1171 (1967).

[25] G. I. Taylor, "Interference Fringes with Feeble Light" P. Camb. Philol. Soc. 15, 114-115 (1909).

[26] A. Einstein, "Strahlungs-Emission und-Absorption nach der Quantentheorie" Verh. Deut. Phys. Ges. 18, 318-323 (1916).

[27] A. Einstein, "Zur Quantentheorie der Strahlung" Mitt. Phys. Ges. Z. 16, 47-62 (1916).

[28] A. Pais, "Einstein and the Quantum Theory" Rev. Mod. Phys. 51, 863-914 (1979).
[29] A. Einstein, "On the Quantum Theory of Radiation" Phys. Z. 18, 121-128 (1917).

[30] A. H. Compton, "A Quantum Theory of the Scattering of X-Rays by Light Elements" Phys. Rev. 21, 483-502 (1923).

[31] M. de Broglie, "Waves and Quanta" C. R. Acad. Sci. (Paris) 177, 507-510 (1923).

[32] S. N. Bose, "Planck's Law and the Light-Quantum Hypothesis" Z. Phys. 26, 178-181 (1924).

[33] E. Fermi, "Sulla Quantizzazione del Gas Perfetto Monoatomico" Rend. Lincei 3, 145-149 (1926).

[34] P. A. M. Dirac, "On the Theory of Quantum Mechanics" P. R. Soc. A. $112,661-677$ (1926).

[35] A. Einstein, "On the Present Status of the Radiation Problem" Phys. Z. 10, 185-193 (1909).

[36] R. Loudon, The Quantum Theory of Light (Clarendon Press, Oxford, 1973).

[37] R. Hanbury Brown, and R. Q. Twiss, "Interferometry of the Intensity Fluctuations in Light. I. Basic Theory: the Correlation Between Photons in Coherent Beams of Radiation" P. Roy. Soc. London 242, 300-324 (1956).

[38] R. Hanbury Brown, and R. Q. Twiss, "Interferometry of the Intensity Fluctuations in Light. II. An Experimental Test of the Theory for Partially Coherent Light" P. Roy. Soc. London 243, 291-319 (1958).

[39] R. Hanbury Brown, and R. Q. Twiss, "Interferometry of the Intensity Fluctuations in Light. III. Applications to Astronomy" P. Roy. Soc. London 248, 199-221 (1958).

[40] R. Hanbury Brown, and R. Q. Twiss, "Interferometry of the Intensity Fluctuations in Light. IV. A Test of an Intensity Interferometer on Sirius A" P. Roy. Soc. London 248, 222-237 (1958).

[41] R. Hanbury Brown, and R. Q. Twiss, "Correlation between Photons in Two Coherent Beams of Light" Nature 177, 27-29 (1956).

[42] R. Hanbury Brown, and R. Q. Twiss, "A Test of a New Type of Stellar Interferometer on Sirius" Nature 178, 1046-1048 (1956).

[43] R. Hanbury Brown, and R. Q. Twiss, "The Question of Correlation Between Photons in Coherent Light Rays" Nature 178, 1447-1448 (1956).

[44] R. Q. Twiss, A. G. Little, and R. Hanbury Brown, "Correlation Between Photons, in Coherent Beams of Light, Detected by a Coincidence Counting Technique" Nature 180, 324-326 (1957).

[45] G. Gorelik, "On the Possibility of Low-Inertia Photometry and Demodulation Analysis of Light" Dokl. Akad. Nauk SSSR+ 58, 45-47 (1947).

[46] A. T. Forrester, R. A. Gudmundsen, and P. 0. Johnson, “Photoelectric Mixing of Incoherent Light" Phys. Rev. 99, 1691-1700 (1955).

[47] W. Feller, An Introduction to Probability Theory and Its Applications : Volume 1 (Third Edition, John Wiley and Sons, New York, 1968).

[48] E. Jakeman, and E. R. Pike, "The Intensity-Fluctuation Distribution of Gaussian Light" J. Phys. A 1 128-138 (1968).

[49] L. Mandel, "Fluctuations of Photon Beams and their Correlations" P. Phys. Soc. Lond. 71, 1037-1048 (1958).

[50] P. L. Kelley, and W. H. Kleiner, "Theory of Electromagnetic Field Measurement and Photoelectron Counting" Phys. Rev. 136, A316334 (1964).

[51] J. P. Gordon, H. J. Zeiger, and C. H. Townes, "Molecular Microwave Oscillator and New Hyperfine Structure in the Microwave Spectrum 
of $\mathrm{NH}_{3}$ " Phys. Rev. 95, 282-284 (1954).

[52] L. Mandel, and E. Wolf, "Comments on a paper by E. Jakeman and E. R. Pike, The Intensity Fluctuation Distribution of Gaussian Light" J. Phys. A 1, 625-627 (1968).

[53] D. Slepian, "Fluctuations of Random Noise Power" Bell Syst. Tech. J. 37, 165-84 (1958).

[54] E. M. Purcell, "The Question of Correlation between Photons in Coherent Light Rays" Nature 178, 1449-1450 (1956).

[55] F. D. Kahn, “0n Photon Coincidences and Hanbury Brown's Interferometer" Opt. Acta 5, 93-100 (1958).

[56] E. R. Pike, "Photon Statistics" Riv. del Nuovo Cimento 1, 277-314 (1969).

[57] R. J. Glauber, Quantum Optics and Electronics, C. DeWitt, A. Blandin, and C. Cohen-Tannoudji, eds., (Gordon and Breach, New York, 1965).

[58] C. Freed, and H. H. Haus, "Photoelectron Statistics Produced by a Laser Operating Below the Threshold of Oscillation" Phys. Rev. Lett. 15, 943-946 (1966).

[59] C. Freed, and H. A. Haus, "Amplitude Noise of Gas Lasers Below and Above the Threshold of Operation" in Physics of Quantum Electronics, P. L. Kelley, B. Lax, and P. E. Tannenwald, eds., 715724 (McGraw Hill, New York, 1966).

[60] F. T. Arecchi, and A. Berné, "High-Order Fluctuations in a SingleMode Laser Field" Phys. Rev. Lett. 16, 32-35 (1966).

[61] A. W. Smith, and J. A. Armstrong, "Observation of Photon-Counting Distribution for Laser Light Below Threshold" Phys. Lett. 19, 650651 (1966).

[62] A. W. Smith, and J. A. Armstrong, "Intensity fluctuations and correlations in a gas laser" in Proceedings of the International Conference on the Physics of Quantum Electronics, P. L. Kelley, B. Lax, and P. E. Tannenwald, eds., 701-705 (McGraw Hill, New York, 1966).

[63] R. F. Chang, R. W. Detenbeck, V. Korenman, and C. O. Alley, "Photon Bunching in a Laser at Threshold" Technical Report No. 689 (University of Maryland, 1967).

[64] M. Lax, and M. Zwanziger, “Exact Photocount Statistics: Lasers near Threshold" Phys. Rev. A. 7, 750-771 (1973).

[65] E. H. Eberhardt, "Multiplier Phototubes for Single-Electron Counting" IEEE T. Nucl. Sci. NS-11, 48-55 (1964).
[66] E. H. Eberhardt, "Multiplier Phototubes as Quantum Counters" Appl. Opt. 6, 161-162 (1967).

[67] E. H. Eberhardt, "Noise Factor Measurements in Multiplier Phototubes" Appl. Opt. 6, 359-360 (1967).

[68] R. Foord, R. Jones, C. J. Oliver, and E. R. Pike, "The Use of Photomultiplier Tubes for Photon Counting" Appl. Opt. 8, 1975-1989 (1969).

[69] A. F. Johnson, R. Jones, T. P. McLean, and E. R. Pike, "Dead-Time Corrections to Photon-Counting Distributions" Phys. Rev. Lett. 16, 589-592 (1966).

[70] C.J. Palin, W. F. Vinen, E. R. Pike, and J. M. Vaughan, “Rayleigh and Brillouin Scattering from Superfluid ${ }^{3} \mathrm{He}-{ }^{4} \mathrm{He}$ mixtures" J. Phys. C. Solid St. Phys 4, L225-L228 (1971).

[71] M. 0. Scully, and W. E. Lamb, Jr., "Quantum Theory of an Optical Maser. I. General Theory" Phys. Rev. 159, 208-226 (1967).

[72] E. R. Pike, and S. Sarkar, The Quantum Theory of Radiation (Clarendon Press, Oxford, 1995)

[73] M. H. Szymanska, A. F. Hughes, and E. R. Pike, "Effect of a Photonic Band Gap on the Threshold and Output Power of Solid-State Lasers and Light-Emitting Diodes" Phys. Rev. Lett. 83, 69-72 (1999).

[74] E. Jakeman, C. J. Oliver, E. R. Pike, M. Lax, and M. Zwanziger, "The Intensity Fluctuation Distribution of Laser Light" J. Phys. A 3 , L52-L55 (1970).

[75] M. Lax, and M. Zwanziger, "Exact Photocount Distributions for Lasers near Threshold" Phys. Rev. Lett. 24, 937-940 (1970).

[76] E. Jakeman, and E. R. Pike, "Spectrum of Clipped Photon-Counting Fluctuations of Gaussian Light" J. Phys. A. 2, 411-412 (1969).

[77] R. Foord, E. Jakeman, C. J. Oliver, E. R. Pike, R. J. Blagrove, E. Wood, and A. R. Peacock, "Determination of Diffusion Coefficients of Haemocyanin at Low Concentration by Intensity Fluctuation Spectroscopy of Scattered Laser Light" Nature 227, 242-245 (1970).

[78] J. G. McWhirter, and E. R. Pike, "On the Numerical Inversion of the Lapace Transform and Similar Fredholm Integral Equations of the First Kind" J. Phys. A. 11, 1729-1745 (1978).

[79] E. R. Pike, and P. Sabatier, Scattering (Academic Press, New York, 2002).

[80] E. R. Pike, "The Malvern Correlator: Case Study in Development" Phys. Technol. 10, 104-109 (1979). 